\title{
Sourcing Competition under Cost Uncertainty and Information Asymmetry
}

\author{
Lusheng Shao \\ Faculty of Business and Economics, University of Melbourne, Victoria 3010, Australia, lusheng.shao@unimelb.edu.au \\ Xiaole $\mathrm{Wu}$ \\ School of Management, Fudan University, Shanghai 20043, China, wuxiaole@fudan.edu.cn \\ Fuqiang Zhang \\ Olin Business School, Washington University in St. Louis, St. Louis, Missouri 63130, fzhang22@wustl.edu
}

\begin{abstract}
Driven by increasing costs in the traditionally-regarded low-cost manufacturing bases (e.g., China), many firms have started to outsource their production to the regions of even lower costs (e.g., Southeast Asia). However, a new environment may involve higher cost uncertainty and severer information asymmetry. Motivated by these observations, we consider a sourcing game where competing firms choose between a supplier with transparent certain cost (type-C supplier) and a supplier with potentially lower but less transparent, uncertain cost (type-U supplier). We characterize the equilibrium of the sourcing game and study how different parameters affect the firms' sourcing strategy and profit performance. First, we find that due to information asymmetry, a large market size can make firms prefer the C-supplier to the U-supplier even if the latter has a lower average cost. Second, reducing the cost uncertainty or improving the signal accuracy of the U-supplier does not necessarily make it more attractive to sourcing firms, which cautions the suppliers when making efforts to mitigate cost uncertainty or improve cost estimation. Third, higher competition intensity makes the diversified sourcing strategy more likely to be adopted under certain conditions. Interestingly, increasing the cost of the C-supplier (e.g., a cost hike in China) may make both sourcing firms better off because it can lead to a new sourcing equilibrium. Finally, this paper shows that the direction of quantity distortion under the optimal competitive mechanism differs from that under the traditional monopolistic setting.
\end{abstract}

Key words: strategic sourcing; information asymmetry; mechanism design; Cournot competition

History: Submitted in January 2019; Revised in July 2019; Accepted in September 2019.

\section{Introduction}

The business media has recently reported that the cost advantage of traditionally-regarded lowcost manufacturing bases is being eroded as a result of a combination of factors. By analyzing the world's 25 leading exporting economies along four key dimensions - manufacturing wages, labor productivity, energy costs, and exchange rates - the Boston Consulting Group finds that China's estimated manufacturing-cost advantage over the U.S. has shrunk to less than 5\%, and Brazil is 
now estimated to be more expensive than much of Western Europe (Sirkin et al. 2014). These facts have driven many companies to rethink their global supply chain strategies.

For product and component sourcing, firms have begun to target Southeast Asia as one attractive sourcing destination. According to The Economist (2015), "the average factory worker in China earns $\$ 27.50$ per day, compared with $\$ 8.60$ in Indonesia and $\$ 6.70$ in Vietnam”. Although Southeast Asia is seemingly attractive in terms of low nominal wages, there is extensive discussion around the drawbacks there. First, many empirical evidences have suggested that the true cost of sourcing from suppliers in this region is far less clear. According to the McKinsey Global Institute, import/export costs are 24\% higher in ASEAN (Association of Southeast Asian Nations) than in China, and the region's customs procedures take $66 \%$ longer than the OECD average (Woetzel et al. 2014). The mediocrity of infrastructure (e.g., roads, power supplies) — compared with Chinaadds costs. Second, as new supply bases, suppliers in Southeast Asia still remain "mysterious" to global buying firms due to the lack of previous interactions. It is believed that prior relationships mitigate information frictions between buyers and suppliers (Costello 2013). ${ }^{1}$ Thus, lack of previous interactions will inevitably aggravate the issue of information asymmetry. As a consequence, firms sourcing from Southeast Asia may find themselves at an informational disadvantage. Such information asymmetry may even represent an important barrier to global trade (Hortacsu et al. 2009).

These features of the suppliers' cost structure in Southeast Asia create a challenge for firms at the crossroad in choosing a sourcing location and designing procurement contract. On the one hand, the cost advantage at well-established manufacturing bases (e.g., China) has been shrinking so that the regions such as Southeast Asia seem to have nominal cost advantage; on the other hand, there is significant cost uncertainty and a lack of transparent information for these less expensive regions. Should a firm select a supplier with transparent, certain cost (referred to as C-supplier), or a supplier with potentially lower, but uncertain cost (referred to as U-supplier who is endowed with more cost information than the buyer)? Such a question may frequently arise given contemporary global business environment. The answer to this question depends on the trade-off between cost efficiency and the potential impact of cost uncertainty and information asymmetry. Such a trade-off can be quite involved because nowadays most firms operate under a global, competitive environment. According to a PwC/Duke Offshoring Research Network survey study, competitive pressures are one of the top four drivers of global sourcing ( $P w C 2010)$. Thus, firms need to take into account their competitor's sourcing strategy when evaluating the trade-off.

\footnotetext{
${ }^{1}$ The empirical study carried out by Costello (2013) measures information asymmetry by using the number of previous interactions between buyers and suppliers.
} 
To our knowledge, the trade-off between cost efficiency and cost information uncertainty/asymmetry in a competitive setting has not been explored in the literature. The goal of this paper is to obtain a better understanding of how such a trade-off drives firms' sourcing strategies. For this purpose, we develop a game-theoretic model where two firms sell substitutable products in the same market. The firms first choose their supplier (either a C-supplier or U-supplier), then offer a contract (or a menu of contracts) to the selected supplier, and finally engage in Cournot (quantity) competition with each other. If a firm chooses a C-supplier, it knows the production cost of the supplier; if a firm chooses a U-supplier, it has a prior belief about the distribution of this supplier's cost, whereas the supplier itself has better information about its own cost (e.g., the U-supplier knows better about the local environment as well as its own production efficiency than the sourcing firm). Depending on the firms' sourcing strategies, we consider three possible sourcing structures, or subgames: $c c$, with both sourcing from C-suppliers; uu, with both sourcing from U-suppliers; and $c u$ (or $u c$ ), with one firm sourcing from a C-supplier and the other sourcing from a U-supplier. This sourcing game can be solved using backward induction: First, we characterize the equilibrium of the competitive mechanism design game under each sourcing structure; then we derive the equilibrium of the sourcing game by comparing the firms' expected profits under the three sourcing structures.

With this model setup, we show that when C-supplier's cost is equal to the average cost of U-supplier, both firms will source from C-suppliers in equilibrium. It is well known that such a result will be reversed if there is no information asymmetry because firms prefer variable cost under Cournot competition (Vives 1999). This implies the presence of information asymmetry has a significant impact on the sourcing equilibrium. In the main analysis of firms' sourcing strategies, we focus on the situation in which the average cost of U-supplier is lower than the cost of Csupplier, which reflects the common belief that suppliers in Southeast Asia have lower production costs on average. Under this practical situation, we analyze how market size, U-supplier's cost uncertainty and signal accuracy, and the competition intensity influence the firms' competitive sourcing strategies.

The main results of this paper are summarized as follows. First, given the sourcing structures $c u$ and $u u$, we find that the firms' profits are not monotonic in the level of cost uncertainty, measured by the gap between the two cost types. That is, reducing cost uncertainty does not necessarily benefit the firms, which suggests that they need to be cautious when investing in uncertaintyreduction activities.

Having analyzed the three sourcing structures, we then characterize the equilibrium of the sourcing game. We show that market size plays an important role in determining the sourcing equilibrium: When the market size is sufficiently small (large), both firms source from U-suppliers 
(C-suppliers); when the market size is medium, firms adopt the diversified sourcing strategies even though they are ex ante symmetric. This is because when sourcing from U-suppliers, firms need to pay the information rent, which may offset the overall benefit from the U-suppliers' nominal cost advantage, and the information rent increases with the market size. Consequently, C-suppliers become more attractive with a larger market size despite their higher average cost. In addition, both cost uncertainty and signal accuracy of the U-supplier have non-monotonic effects on the sourcing equilibrium. In other words, reducing cost uncertainty or improving cost estimation may not necessarily imply that U-suppliers will be more likely chosen by the firms. We also show that intensified competition can favor either C-suppliers or U-suppliers, depending on the current competition level. More interestingly, we find that both firms can be better off when the C-supplier's cost increases. This implies that, contrary to our intuition, the rising cost of Chinese suppliers may benefit all the sourcing firms.

Finally, we find that in subgames $c u$ and $u c$ the firms distort downward the order quantities when facing both high-cost and low-cost U-suppliers. This result is in contrast with the standard result in the literature that "no distortion at the top but downward distortion at the bottom" for a single supply chain, highlighting the important implication of competition in the optimal contract design with asymmetric information.

The remainder of this paper is organized as follows. Section 2 reviews the related literature, and Section 3 sets up the model. We analyze the mechanism design subgames in Sections 4 and 5 . The first stage's sourcing game is studied in Section 6. Finally, we conclude in Section 7. All proofs are presented in the appendices.

\section{Literature Review}

This paper studies a sourcing game where the firms engage in Cournot competition and the suppliers may have private cost information. There is a stream of research that studies contracting problems under asymmetric cost information in supply chain settings. The representative studies include, for example, Corbett and de Groote (2000), Corbett (2001), Ha (2001), Corbett et al. (2004), Cachon and Zhang (2006), Lutze and Özer (2008), Zhang (2010), Özer and Raz (2011), Bolandifar et al. (2018), and Hu and Qi (2018). Most of these studies focus on screening models where the uninformed player moves first by offering a menu of contracts to elicit the informed player's private cost information. Similarly, there is a line of research that considers asymmetric information in other dimensions and problem settings. For instance, Cachon and Lariviere (2001), Özer and Wei (2006), Burnetas et al. (2007), and Oh and Özer (2013) consider asymmetric demand information. Yang et al. (2009) consider asymmetric information regarding supply disruption risk. Kostamis and Duenyas (2011) examine a situation where a downstream firm (i.e., an OEM) possesses two-dimensional private information: demand and cost. Kayis et al. (2013) examine the 
problem of delegation versus control when a buyer purchases two components that are each produced by different privately informed suppliers. Li et al. (2015b) study supply contract design for competing suppliers under asymmetric demand information with a focus on the trade-off between cost advantage and information rent. Most of the above studies consider either a one-to-one or one-to-many supply chain structure. In contrast, we consider competing supply chains with asymmetric cost information. That is, we extend the supply contract design problem with information asymmetry to a competitive setting.

Our model studies a setting with competing supply chains, and hence is related to the literature on chain-to-chain competition and oligopolistic competition. Under chain-to-chain competition, some of the studies in marketing explore the effect of channel structure and product differentiation on channel performance (McGuire and Staelin 1983, Moorthy 1988, Choi 1996). The economics literature studies the managerial incentives in oligopolistic settings; see the seminal paper by Fershtman and Judd (1987). Notably, the above literature studies games with full information. Several recent papers have started to incorporate asymmetric information into models for various problem settings. In operations management, Ha and Tong (2008) investigate the contract design and information-sharing decisions in two competing supply chains. Ha et al. (2011) study the incentive of vertical information sharing when the upstream firms' production technologies exhibit diseconomies of scale. Etro and Cella (2013) study incentive contracts for competing firms that engage in R\&D activities by hiring privately informed managers. In Li et al. (2015a), a supplier may encroach and compete with a reseller. The paper characterizes the supplier's optimal nonlinear contract when the reseller has better market size information. They find that due to the supplier's potential subsequent encroachment and competition, the quantities sold through the reselling channel can distort upward. Our paper also studies a competitive setting. However, the problem we focus on is different: We examine how the interaction of downstream competition and asymmetric information affects firms' contract design and supplier-selection decisions. As a result, we find some results that have not been reported in the existing literature.

Another closely related literature studies firms' sourcing and/or outsourcing strategies under competition. Many papers have documented the benefit of outsourcing in comparison with insourcing (Cachon and Harker 2002, Gilbert et al. 2006). As opposed to these findings, Feng and Lu (2012) show that low-cost outsourcing may lead to a win-lose situation where suppliers gain while manufacturers lose. Specifically, they investigate competing manufacturers' sourcing decisions by using a multi-unit bilateral bargaining framework. Wu and Zhang (2014) analyze a multi-stage sourcing game for competing firms that choose either responsive sourcing or efficient sourcing. Their focus is on how demand information accuracy and correlation, as well as the deterministic cost difference, affect firms' equilibrium sourcing strategies. Our paper differs from this literature 
in that we are concerned with the effect of cost information uncertainty/asymmetry and cost difference on firms' contracting and sourcing decisions; the sourcing cost from the supplier with private information is the outcome of a mechanism design problem under competition.

Finally, there is an economics literature on competitive mechanism design. For the representative papers, please refer to Peters and Severinov (1997), Epstein and Peters (1999), and McAfee (1999). This stream of literature considers a setting where multiple principals compete in selling goods to a common pool of agents by designing mechanisms. The focus is on characterizing the equilibrium mechanism choices for the principals and the equilibrium bidding strategies for the agents. Both the model setting and results from our paper are quite different: The principals are buyers instead of sellers; in addition, the principals make supplier selection decisions initially, and then offer an exclusive contract or contract menu to their own agent (i.e., supplier) by taking into account the competitor's optimal mechanism.

\section{The Model}

Consider two firms selling substitutable products in the same market. The firms can be either manufacturers or retailers. For easy reference, we will call the firms manufacturers and use "she" to refer to a manufacturer. Suppose the manufacturers engage in Cournot competition, which is commonly used in the literature (see, e.g., Ha and Tong 2008, Ha et al. 2011, Wu and Zhang 2014). The market-clearing price is $p_{k}=A-q_{k}-\gamma q_{3-k}, k=1,2$, where $A$ is the market size, $q_{k}$ is manufacturer $k$ 's output quantity, and $\gamma \in[0,1]$ measures the competition intensity.

We study a procurement setting where the manufacturers source their product (or a critical input) from exclusive suppliers. We will use "it" to refer to a supplier. In the procurement context, two of the most important decisions facing the manufacturers include supplier selection and procurement contract design. Motivated by the recent debate about the manufacturing costs in different regions, we consider two types of suppliers: the suppliers with a transparent cost $c$ (i.e., C-suppliers) and those with a non-transparent, uncertain cost $\hat{c}$ (i.e., U-suppliers). One possible interpretation is that the $\mathrm{C}$-suppliers are from well-established manufacturing bases with greater transparency (e.g., China) while the U-suppliers are located in regions with potentially lower costs that are less known to outsiders (e.g., Southeast Asia).

We are interested in situations where each manufacturer only contracts with one supplier. Sole sourcing provides multiple well-known benefits for an organization, such as reductions in product variation, training required, and cost of quality (Dipak 2002). When buyers have special product implementation needs, it takes time to develop a supplier and effectively convey all the technical specifications so as to make sure all the guidelines are strictly followed by suppliers. Thus, in situations where developing or managing suppliers is very costly, it might not be worthwhile sourcing from multiple suppliers. 
The sequence of the multi-stage sourcing game under Cournot competition is as follows: First, the manufacturers simultaneously decide which type of suppliers to source from. Denote a C-supplier by $S_{c}$, and a U-supplier by $S_{u}$. Based on the manufacturers' supplier selection decisions, there are four possible sourcing structures: $\left(S_{c}, S_{c}\right),\left(S_{c}, S_{u}\right),\left(S_{u}, S_{c}\right)$, and $\left(S_{u}, S_{u}\right)$. These correspond to four subgames: $c c, c u, u c$, and $u u$. In reality, it is not uncommon that there are uncertainties associated with the suppliers' production costs. However, the U-supplier's capability is generally more uncertain and less known to outsiders. Therefore, we normalize the cost uncertainty of the C-supplier to zero for analytical transparency, while the manufacturers have a prior belief about the distribution of U-supplier's cost (details will be provided later in this section).

Second, the manufacturers develop their selected suppliers' technological capability in terms of product quality, reliability, or delivery performance. After supplier development, the U-supplier, after knowing the details of the manufacturer's needs about the product, obtains a better understanding of its own production cost. The U-supplier's better information compared to the manufacturer's is modeled by a cost signal; that is, the U-supplier receives a cost signal $s$ about its unit cost, which is assumed to be either high $\left(s_{h}\right)$ or low $\left(s_{l}\right)$. The signal is the U-supplier's private information and is unobservable to the manufacturer. Thus, there exists information asymmetry between the manufacturer and the U-supplier. ${ }^{2}$

Third, under each subgame, the manufacturers offer a procurement contract to their exclusive supplier. If a manufacturer chooses a U-supplier who holds private cost signal, then she offers a menu of contracts to screen the type of the chosen supplier. If a manufacturer chooses a Csupplier, she offers a take-it-or-leave-it contract to the supplier. Note that the contract terms in one supply chain are not observable to the competing chain. Each manufacturer forms a belief about the opponent's strategy (instead of observing it) to obtain the best response functions, and the equilibrium contract terms for both manufacturers are derived by jointly solving the best response functions.

Lastly, the C-supplier decides whether to accept the contract offered by its buyer, and/or the U-supplier decides which contract to select from the contract menu. Then, the manufacturers' total outputs determine the market-clearing price, and the manufacturers collect their profits. We assume all parties are risk neutral in the sourcing game. Without losing generality, the reservation profit (i.e., the best outside option) for each party is normalized to zero.

In the following, we specify the detailed cost information structure and the updating mechanism.

\footnotetext{
${ }^{2}$ Both the manufacturer and the U-supplier may update their belief about the supplier's cost during the development phase. However, the information improvement at the U-supplier side could be much more significant and U-supplier would still have better knowledge about the local environment, the costs of raw materials, its own production efficiency, and so on. Thus, information asymmetry in this context captures the U-supplier's superior cost information.
} 
Prior probabilities of U-supplier's cost: From the manufacturers' perspective, the U-supplier's ex ante cost $\hat{c}$ is drawn from a two-point distribution with $\operatorname{Pr}\left(\hat{c}=c_{l}\right)=\operatorname{Pr}\left(\hat{c}=c_{h}\right)=1 / 2$, where $c_{h}>c_{l}$. In the later comparative statics analysis, we define $c_{h}=\mu+\delta$ and $c_{l}=\mu-\delta$, where $\delta$ measures the ex ante cost uncertainty. This assumption is for ease of exposition; the qualitative results will remain unchanged if we extend to a multi-point distribution or a weight other than $1 / 2$; please see Appendix $\mathrm{C}$ for the possible extension to multi-point distributions.

Conditional probabilities given signal quality: Suppose the probabilities of signals conditional on costs are

$$
\operatorname{Pr}\left(s_{l} \mid c_{l}\right)=\operatorname{Pr}\left(s_{h} \mid c_{h}\right)=\alpha \quad \text { and } \operatorname{Pr}\left(s_{l} \mid c_{h}\right)=\operatorname{Pr}\left(s_{h} \mid c_{l}\right)=1-\alpha,
$$

where $\alpha \in(1 / 2,1]$ measures the signal quality or accuracy. When $\alpha=1$, the signal reveals perfect information. When $\alpha=1 / 2$, the signal does not convey any information and essentially there is no information asymmetry between the U-supplier and the manufacturer.

Using Bayes' Theorem, we can calculate the posterior probabilities based on the above prior and conditional probabilities. We have

$$
\operatorname{Pr}\left(c_{l} \mid s_{l}\right)=\frac{\operatorname{Pr}\left(s_{l} \mid c_{l}\right) \operatorname{Pr}\left(c_{l}\right)}{\operatorname{Pr}\left(s_{l} \mid c_{l}\right) \operatorname{Pr}\left(c_{l}\right)+\operatorname{Pr}\left(s_{l} \mid c_{h}\right) \operatorname{Pr}\left(c_{h}\right)}=\alpha,
$$

and similarly, $\operatorname{Pr}\left(c_{h} \mid s_{h}\right)=\alpha, \quad \operatorname{Pr}\left(c_{h} \mid s_{l}\right)=1-\alpha, \quad \operatorname{Pr}\left(c_{l} \mid s_{h}\right)=1-\alpha$. We can also calculate the marginal probabilities of the signals as $\operatorname{Pr}\left(s_{l}\right)=\operatorname{Pr}\left(s_{h}\right)=1 / 2$.

For convenience, we let $\hat{C} \equiv \hat{c} \mid s$ denote the U-supplier's cost conditional on the received signal $s$, which we term the conditional cost. Since the signal may be high or low, the conditional cost $\hat{C}$ may take the following two values,

$$
C_{k}=c_{l} \operatorname{Pr}\left(c_{l} \mid s_{k}\right)+c_{h} \operatorname{Pr}\left(c_{h} \mid s_{k}\right), \quad \text { for } k=l, h .
$$

Note that in our model, even though the U-supplier observes its private signal, it still faces cost uncertainty. Therefore, $C_{k}$ essentially measures the expected cost conditional on the signal $s_{k}$. Specifically, $C_{l}=\alpha c_{l}+(1-\alpha) c_{h}$ and $C_{h}=\alpha c_{h}+(1-\alpha) c_{l}$. Thus we have $C_{h}-C_{l}=(2 \alpha-1)\left(c_{h}-c_{l}\right)>$ 0 , implying that a higher signal translates to a higher conditional cost. We can also show that the expected conditional cost is $E[\hat{C}]=\left(C_{h}+C_{l}\right) / 2=\left(c_{h}+c_{l}\right) / 2$.

Correlation between signals: If both manufacturers choose U-suppliers, the U-suppliers' costs may be correlated. We model the cost correlation by explicitly specifying their joint distributions as follows:

$$
\begin{aligned}
& \operatorname{Pr}\left(\hat{c}_{i}=c_{l}, \hat{c}_{j}=c_{l}\right)=\operatorname{Pr}\left(\hat{c}_{i}=c_{h}, \hat{c}_{j}=c_{h}\right)=\tau, \\
& \operatorname{Pr}\left(\hat{c}_{i}=c_{l}, \hat{c}_{j}=c_{h}\right)=\operatorname{Pr}\left(\hat{c}_{i}=c_{h}, \hat{c}_{j}=c_{l}\right)=\frac{1}{2}-\tau .
\end{aligned}
$$


It follows that the correlation coefficient between $\hat{c}_{i}$ and $\hat{c}_{j}$ is $4 \tau-1$, where a greater value of $\tau$ indicates a stronger correlation. In particular, $\tau=1 / 2$ corresponds to perfect correlation and $\tau=1 / 4$ represents the case with independent costs. Since the U-suppliers are located in the same region, we assume $1 / 4<\tau \leq 1 / 2$, i.e., from the manufacturers' perspective, their costs are positively correlated. We also assume U-suppliers' signals are conditionally independent given their costs, i.e., $\operatorname{Pr}\left(s_{i}=s_{k}, s_{j}=s_{k^{\prime}} \mid \hat{c}_{i}=c_{m}, \hat{c}_{j}=c_{m^{\prime}}\right)=\operatorname{Pr}\left(s_{k} \mid c_{m}\right) \operatorname{Pr}\left(s_{k^{\prime}} \mid c_{m^{\prime}}\right)$ for any $k, k^{\prime}, m, m^{\prime} \in\{l, h\}$.

Now we can derive the joint distribution of U-suppliers' signals as follows

$$
\operatorname{Pr}\left(s_{i}=s_{l}, s_{j}=s_{l}\right)=\tau(2 \alpha-1)^{2}+\alpha(1-\alpha) .
$$

Similarly, we obtain

$$
\begin{aligned}
& \operatorname{Pr}\left(s_{i}=s_{h}, s_{j}=s_{h}\right)=\tau(2 \alpha-1)^{2}+\alpha(1-\alpha), \\
& \operatorname{Pr}\left(s_{i}=s_{l}, s_{j}=s_{h}\right)=\operatorname{Pr}\left(s_{i}=s_{h}, s_{j}=s_{l}\right)=-\tau(2 \alpha-1)^{2}+\left[\alpha^{2}+(1-\alpha)^{2}\right] / 2 .
\end{aligned}
$$

For convenience, define $\beta \equiv \tau(2 \alpha-1)^{2}+\alpha(1-\alpha)$. It can be readily shown that $1 / 4<\beta \leq 1 / 2$ for $\alpha \in(1 / 2,1]$ and $\tau \in(1 / 4,1 / 2]$. We can then use $\beta$ to represent the joint probabilities of U-suppliers' signals below:

$$
\begin{aligned}
& \operatorname{Pr}\left(s_{i}=s_{l}, s_{j}=s_{l}\right)=\operatorname{Pr}\left(s_{i}=s_{h}, s_{j}=s_{h}\right)=\beta, \\
& \operatorname{Pr}\left(s_{i}=s_{l}, s_{j}=s_{h}\right)=\operatorname{Pr}\left(s_{i}=s_{h}, s_{j}=s_{l}\right)=\frac{1}{2}-\beta .
\end{aligned}
$$

In the following, by backward induction we first analyze the mechanism design subgames in Sections 4 and 5, and then the first stage's sourcing game in Section 6 .

\section{Analysis for Subgame $c u(u c)$}

We start with the case where the manufacturers use different types of suppliers. This case includes the subgames $c u$ and $u c$. By symmetry, we only need to focus on the subgame $c u$. Suppose, without loss of generality, manufacturer $i$ sources from a C-supplier, while manufacturer $j$ sources from a U-supplier. Manufacturer $i$ offers a contract $\left(q_{i}, T_{i}\right)$ to her chosen supplier $i$, while manufacturer $j$ offers a contract menu $\left(q_{j l}, T_{j l}, q_{j h}, T_{j h}\right)$ to her supplier $j$. Supplier $j$, with its private cost signal, may select a contract from the menu. Then, the two manufacturers engage in quantity competition by selling their products to the market. The contract terms selected in one supply chain are not observable to the firms in the other supply chain because they are typically important confidential information in a competitive business environment. We have proved in Appendix D that during the selling stage, the manufacturers will sell all the products they have ordered to the market, i.e., they will not withhold inventory. This is because when designing the contract terms, the manufacturers 
can anticipate the needed selling quantities in the third stage conditional on U-supplier's cost type, and hence they will not order more than needed since the transfer prices to the supplier increase in order quantities.

Let $\Pi_{k}(k=i, j)$ denote manufacturer $k^{\prime} s$ expected profit and $\pi_{k}(k=i, j)$ denote supplier $k^{\prime} s$ expected profit. Given manufacturer $j$ 's contract menu, we first look at manufacturer $i$ 's best response in choosing the optimal contract $\left(q_{i}, T_{i}\right)$. Note that manufacturer $i$ does not know supplier $j$ 's actual signal, so in the subgame $c u$, manufacturer $i$ has to take expectation over supplier $j$ 's signal. Since manufacturer $i$ knows supplier $i$ 's cost, she can leave supplier $i$ the reservation profit only, which is normalized to zero. In response to manufacturer $j$ 's contract menu $\left(q_{j l}, T_{j l}, q_{j h}, T_{j h}\right)$, manufacturer $i$ 's problem is formulated as follows,

$$
\max _{T_{i}, q_{i}} \quad \Pi_{i}=\frac{1}{2}\left(A-q_{i}-\gamma q_{j l}\right) q_{i}+\frac{1}{2}\left(A-q_{i}-\gamma q_{j h}\right) q_{i}-T_{i}
$$

subject to supplier $i^{\prime}$ s participation constraint $T_{i}-c q_{i} \geq 0$, which guarantees that supplier $i$ earns no less than its reservation profit.

Now consider manufacturer $j$ 's problem. Since supplier $j$ has a private cost signal $s_{j}$, manufacturer $j$ offers two options on the menu, $\left(q_{j l}, T_{j l}\right)$ and $\left(q_{j h}, T_{j h}\right)$, to elicit supplier $j$ 's private cost signal information. By the Revelation Principle (Dasgupta et al. 1979, Myerson 1979), we focus on the truth-telling mechanism in which supplier $j$ will truthfully reveal its type by choosing the corresponding contract.

Given manufacturer $i$ 's contract $\left(q_{i}, T_{i}\right)$, we formulate manufacturer $j$ 's mechanism design problem in the following:

$$
\max _{q_{j l}, T_{j l}, q_{j h}, T_{j h}} \Pi_{j}=\frac{1}{2}\left[\left(A-\gamma q_{i}-q_{j l}\right) q_{j l}-T_{j l}\right]+\frac{1}{2}\left[\left(A-\gamma q_{i}-q_{j h}\right) q_{j h}-T_{j h}\right],
$$

subject to the individual rationality (IR) and the incentive compatibility (IC) constraints,

$$
\begin{aligned}
& \text { IRs: } T_{j k}-C_{k} q_{j k} \geq 0, \quad \forall k=l, h, \\
& \text { ICs: } T_{j k}-C_{k} q_{j k} \geq T_{j k^{\prime}}-C_{k} q_{j k^{\prime}}, \quad \forall k, k^{\prime}=l, h \text { and } k \neq k^{\prime},
\end{aligned}
$$

where $C_{k}$ is given in Equation (1). Solving the above problems for manufacturer $i$ in Equation (2) and manufacturer $j$ in Equation (3), we obtain the equilibrium in Proposition 1.

Proposition 1. Consider the contract design subgame cu in which manufacturer $i$ selects a $C$-supplier and manufacturer $j$ selects a U-supplier who has private cost signal. 
(i) There exists a unique Bayesian Nash Equilibrium (BNE) $\left\{\left(q_{i}^{c u}, T_{i}^{c u}\right),\left(q_{j l}^{c u}, T_{j l}^{c u}, q_{j h}^{c u}, T_{j h}^{c u}\right)\right\}$, where

$$
\begin{aligned}
q_{i}^{c u} & =\frac{A-c}{2+\gamma}+\frac{\gamma\left(C_{h}-c\right)}{4-\gamma^{2}}, \quad T_{i}^{c u}=c q_{i}^{c u}, \\
q_{j l}^{c u} & =\frac{A-C_{l}}{2+\gamma}+\frac{\gamma(2-\gamma)\left(C_{h}-C_{l}\right)+2 \gamma\left(c-C_{h}\right)}{2\left(4-\gamma^{2}\right)}, \quad T_{j l}^{c u}=C_{l} q_{j l}^{c u}+\left(C_{h}-C_{l}\right) q_{j h}^{c u}, \\
q_{j h}^{c u} & =\frac{A-C_{h}}{2+\gamma}+\frac{\left(\gamma^{2}-4\right)\left(C_{h}-C_{l}\right)+2 \gamma\left(c-C_{h}\right)}{2\left(4-\gamma^{2}\right)}, \quad T_{j h}^{c u}=C_{h} q_{j h}^{c u} .
\end{aligned}
$$

(ii) The manufacturers' profits in equilibrium are $\Pi_{i}^{c u}=\left(q_{i}^{c u}\right)^{2}$ and $\Pi_{j}^{c u}=\frac{1}{2}\left(q_{j l}^{c u}\right)^{2}+\frac{1}{2}\left(q_{j h}^{c u}\right)^{2}$. Supplier i's profit is $\pi_{i}^{c u}=0$, and supplier j's profit is $\pi_{j}^{c u}=\frac{1}{2}\left(C_{h}-C_{l}\right) q_{j h}^{c u}$.

We begin by noting that the condition to ensure a positive order quantity from the high-cost type supplier is $A>C_{h}+\frac{\left(4-\gamma^{2}\right)\left(C_{h}-C_{l}\right)+2 \gamma\left(C_{h}-c\right)}{2(2-\gamma)}$. We obtain from Proposition 1 that manufacturer $j$ 's expected order quantity is $E\left[q_{j}^{c u}\right]=\frac{A-C_{h}}{2+\gamma}+\frac{\gamma\left(c-C_{h}\right)}{4-\gamma^{2}}$. Also note that manufacturer $i$ 's order quantity is $q_{i}^{c u}=\frac{A-c}{2+\gamma}+\frac{\gamma\left(C_{h}-c\right)}{4-\gamma^{2}}$. These equilibrium quantities match those in a Cournot competition model with asymmetric manufacturers whose costs are $c$ and $C_{h}$, respectively. This suggests that, from manufacturer $i$ 's perspective, it seems as if she is competing with a manufacturer whose expected virtual sourcing cost is $C_{h}$. Since manufacturer $i$ does not know supplier $j$ 's cost signal, she has to make an estimation on $s_{j}$. Anticipating that manufacturer $j$ will screen the type of supplier $j$ by paying an information rent, in equilibrium manufacturer $i$ 's estimation of $\hat{C}_{j}$ is supplier $j$ 's high-cost type, $C_{h}$.

Proposition 1 also shows that supplier $i$ attains a profit equal to its reservation profit, which is zero. The high-cost type of supplier $j$ does not receive any information rent, while the low-cost type receives a positive information rent $\left(C_{h}-C_{l}\right) q_{j h}^{c u}$. In expectation, supplier $j$ makes a profit of $\left(C_{h}-C_{l}\right) q_{j h}^{c u} / 2$. We observe that supplier $j$ 's profit increases in $A$, implying that more information rents have to be relinquished to supplier $j$ when the market size is larger.

\subsection{Impact of Information Asymmetry}

In a single chain setting (i.e., without competition), it is well known that information asymmetry will cause the manufacturer to downward-distort at the bottom (order less when facing the least efficient supplier), but not to distort at the top (when facing the most efficient type), compared to the symmetric information case. How would this result change in our competitive setting? To investigate the role of information asymmetry between manufacturer $j$ and supplier $j$, we have examined an auxiliary benchmark case with symmetric information; that is, supplier $j$ 's cost signal is known to manufacturer $j$ but not to the firms in the other supply chain, and supplier $j$ 's actual cost is still uncertain. To save space, we relegate the detailed analysis for the benchmark to 
Appendix A. Note that all the notation for the benchmark has a bar (i.e., -) on top. Lemma A.1 in Appendix A summarizes the equilibrium results under $c u$ in the benchmark case.

By comparing Proposition 1 with Lemma A.1, we are able to derive the impact of information asymmetry on manufacturers' competition. We begin with the comparison in terms of order quantities. For manufacturer $i$, we obtain

$$
q_{i}^{c u}-\bar{q}_{i}^{c u}=\frac{\gamma\left(C_{h}-C_{l}\right)}{2\left(4-\gamma^{2}\right)}>0
$$

For manufacturer $j$ 's order quantities, we have

$$
q_{j l}^{c u}-\bar{q}_{j l}^{c u}=-\frac{\gamma^{2}\left(C_{h}-C_{l}\right)}{4\left(4-\gamma^{2}\right)}<0, \quad q_{j h}^{c u}-\bar{q}_{j h}^{c u}=-\frac{\left(8-\gamma^{2}\right)\left(C_{h}-C_{l}\right)}{4\left(4-\gamma^{2}\right)}<0
$$

The above comparisons suggest that, under competition, manufacturer $i^{\prime} s$ quantity is distorted upward, while manufacturer $j^{\prime} s$ quantities for both high-cost type and low-cost type are distorted downward. It is understandable that with information asymmetry, manufacturer $j$ should order less from the high-cost supplier in order to prevent the low-cost supplier from mimicking the high-cost supplier. In the competitive setting, manufacturer $i^{\prime} s$ quantity decision depends on manufacturer $j^{\prime} s$ expected quantity. In response to manufacturer $j^{\prime} s$ downward quantity distortion when facing a high-cost type, manufacturer $i$ would take advantage of such a behavior by inflating her own order quantity. This in turn drives manufacturer $j$ to distort its quantity downward even when facing a low-cost type supplier. Such a result is in contrast with the standard result "no distortion at the top (when facing a low-cost type) but downward distortion at the bottom (when facing a high-cost type)" that occurs in a single supply chain.

The impact of the information asymmetry on the two manufacturers' profits are relatively intuitive. We can show $\Pi_{i}^{c u}>\bar{\Pi}_{i}^{c u}$ and $\Pi_{j}^{c u}<\bar{\Pi}_{j}^{c u}$. That is, due to information asymmetry, manufacturer $j$ has to pay information rent to supplier $j$ and hence becomes worse off. However, manufacturer $i$ benefits from the information asymmetry between manufacturer $j$ and supplier $j$.

We next investigate how the equilibrium outcomes are affected by model parameters. As defined before, $c_{h}=\mu+\delta$ and $c_{l}=\mu-\delta$, where $\delta$ captures the cost uncertainty of a U-supplier, and a larger $\delta$ implies a higher cost uncertainty. Hereafter, we work with $\mu$ and $\delta$ instead of $c_{h}$ and $c_{l}$. Then the conditional costs are $C_{h}=\mu+(2 \alpha-1) \delta$ and $C_{l}=\mu-(2 \alpha-1) \delta$. Plugging these expressions into Proposition 1, we can represent the equilibrium quantities and profits by using $\mu$ and $\delta$. To avoid triviality, we assume $A>\max \left[(2 c-\gamma(\mu+(2 \alpha-1) \delta)) /(2-\gamma),\left(2 \mu+\left(6-\gamma^{2}\right)(2 \alpha-1) \delta-\gamma c\right) /(2-\gamma)\right]$ such that all the equilibrium quantities are positive. 


\subsection{Effect of Cost Uncertainty}

Cost uncertainty plays an important role in determining equilibrium outcomes. Understanding the impact of cost uncertainty could be useful because manufacturers may need to evaluate the benefits when making investments to reduce cost uncertainty. Define

$$
\delta_{m}^{c u}=\frac{2(2(A-\mu)-\gamma(A-c))}{(2 \alpha-1)\left(\gamma^{4}-8 \gamma^{2}+20\right)}
$$

Proposition 2. Under the subgame cu where the U-supplier (i.e., supplier $j$ ) has private cost information, we have: (i) $\frac{\partial \Pi_{i}^{c u}}{\partial \delta}>0$; (ii) $\frac{\partial \Pi_{j}^{c u}}{\partial \delta}<0$ for $\delta<\delta_{m}^{c u}$, and $\frac{\partial \Pi_{j}^{c u}}{\partial \delta}>0$ for $\delta>\delta_{m}^{c u} \cdot 3$

The above proposition shows that the manufacturer who selects a C-supplier benefits from increased cost uncertainty. As discussed earlier, with information asymmetry, manufacturer $i^{\prime} \mathrm{s}$ order quantity is distorted upward, and by Equation (7), the distortion level increases in $\delta$. Thus, larger cost uncertainty leads to greater distortion, and as a result manufacturer $i$ can benefit more.

However, Proposition 2 (ii) shows the non-monotonic effect of $\delta$ on manufacturer $j^{\prime}$ s profit. This non-monotonicity result is driven by two opposite effects of increasing cost uncertainty on manufacturer $j^{\prime} s$ profitability: The result without information asymmetry suggests that a larger cost uncertainty always benefits the manufacturer who selects a U-supplier (i.e., $\bar{\Pi}_{j}^{c u}$ increases in $\delta$ ), and this is the positive effect of increasing cost uncertainty for manufacturer $j$. However, the presence of information asymmetry forces manufacturer $j$ to distort the quantities downward, and by Equation (8) the distortion is greater for larger $\delta$. This is the negative effect of increasing $\delta$. Aggregating the positive and negative effects, overall increasing $\delta$ can make manufacturer $j$ either better off or worse off, depending on the market size and the initial uncertainty level. If the market size is sufficiently large (which implies $\delta<\delta_{m}^{c u}$ always holds) or the initial uncertainty is low, the negative effect of information asymmetry is large due to high information rent paid or the positive effect out of more variable costs is small; overall increasing $\delta$ hurts manufacturer $j$, i.e., making an effort to reduce uncertainty is beneficial. In the rest cases, cost uncertainty reduction hurts manufacturer $j$.

\subsection{Effect of Signal Quality}

In this subsection, we examine the effect of signal quality $\alpha$ on the equilibrium outcome. Since the signal is private information of the U-supplier, a more accurate signal implies greater information asymmetry between the U-supplier and the manufacturer who sources from it. We find that $q_{j l}^{c u}$ increases in $\alpha$ but $q_{j h}^{c u}$ decreases in $\alpha$. That is, as $\alpha$ increases, the manufacturer choosing a U-supplier

\footnotetext{
${ }^{3}$ The comparative statics results about $\delta$ and $\alpha$ in Sections 4 and 5 are derived given a sourcing structure. We have checked numerically that such results also exist when imposing the equilibrium conditions for a specific sourcing structure in Proposition 6.
} 
orders more from a low-cost type but less from a high-cost type. This implies a more accurate signal in chain $j$ allows manufacturer $j$ to design a menu of quantities that are more tailored to the corresponding types. The effect of signal accuracy on each manufacturer's profit is characterized below. Define $\hat{\alpha}_{m}=\frac{1}{2}+\frac{2(A-\mu)-\gamma(A-c)}{\delta\left(\gamma^{4}-8 \gamma^{2}+20\right)}$.

Proposition 3. Under the subgame cu where the U-supplier (i.e., supplier $j$ ) has private cost information, we have: (i) $\frac{\partial \Pi_{i}^{c u}}{\partial \alpha}>0$; (ii) $\frac{\partial \Pi_{j}^{c u}}{\partial \alpha}<0$ for $\alpha<\hat{\alpha}_{m}$, and $\frac{\partial \Pi_{j}^{c u}}{\partial \alpha}>0$ for $\alpha>\hat{\alpha}_{m}$.

Not surprisingly, a greater level of information asymmetry in chain $j$ benefits manufacturer $i$. However, its impact on the profit of manufacturer $j$ is less clear. On the one hand, a greater $\alpha$ value implies more accurate cost information in chain $j$, and manufacturer $j$ can design a menu of quantities that are more tailored to the corresponding types. This is the positive effect of a larger $\alpha$ on manufacturer $j^{\prime} s$ profit. On the other hand, a larger $\alpha$ implies manufacturer $j$ is at a more disadvantageous position compared to supplier $j$ in term of cost information, and thus hurts manufacturer $j$ more. This negative effect of a larger $\alpha$ combined with the former positive effect may lead to the non-monotonic impact of $\alpha$, depending on the market size $A$ and $\alpha$. For sufficiently large $A$ (which implies $\alpha \leq \hat{\alpha}_{m}$ always holds), the negative informational disadvantage effect is amplified, and thus a larger $\alpha$ always decreases manufacturer $j$ 's profit. For market size that is not too large, the overall effect of increasing $\alpha$ can be either way. For small $\alpha$ values, the information accuracy effect on chain $j$ of a larger $\alpha$ is limited, and the negative information disadvantageous effect on manufacturer $j$ dominates. But for large $\alpha$ values, the marginal increase of informational disadvantage due to a larger $\alpha$ is limited compared to information accuracy improvement, and hence manufacturer $j$ benefits from a larger $\alpha$.

\section{Analysis for Subgame uu (cc)}

We now consider the symmetric mechanism design subgames. We focus on the subgame $u u$ in which both manufacturers purchase from U-suppliers, since the subgame $c c$ is a special case of the subgame $u u$. To elicit private information, each manufacturer offers a contract menu to her exclusive supplier. After receiving the manufacturer's contract menu, each supplier determines which contract to choose from the menu. Again, we focus on the truth-telling mechanism in which the suppliers truthfully reveal their types when selecting a contract from the offered menu.

Given manufacturer $j$ 's contract menu $\left(q_{j l}, T_{j l}, q_{j h}, T_{j h}\right)$, manufacturer $i$ maximizes her expected profit by designing a contract menu $\left(q_{i l}, T_{i l}, q_{i h}, T_{i h}\right)$. We now formulate manufacturer $i$ 's best response problem as follows:

$$
\begin{aligned}
\max _{q_{i l}, T_{i l}, q_{i h}, T_{i h}} \Pi_{i}= & \beta\left[\left(A-q_{i l}-\gamma q_{j l}\right) q_{i l}-T_{i l}+\left(A-q_{i h}-\gamma q_{j h}\right) q_{i h}-T_{i h}\right] \\
& +\left(\frac{1}{2}-\beta\right)\left[\left(A-q_{i l}-\gamma q_{j h}\right) q_{i l}-T_{i l}+\left(A-q_{i h}-\gamma q_{j l}\right) q_{i h}-T_{i h}\right],
\end{aligned}
$$


subject to the individual rationality and the incentive compatibility constraints:

$$
\begin{aligned}
& \text { IRs: } T_{i k}-C_{k} q_{i k} \geq 0, \quad \forall k=l, h, \\
& \text { ICs: } T_{i k}-C_{k} q_{i k} \geq T_{i k^{\prime}}-C_{k} q_{i k^{\prime}}, \quad \forall k, k^{\prime}=l, h \text { and } k \neq k^{\prime} .
\end{aligned}
$$

By symmetry, manufacturer $j$ 's best response problem given manufacturer $i$ 's contract menu can be formulated similarly. Solving the above problems for both manufacturers, we obtain the equilibrium for the subgame $u$ in Proposition 4.

Proposition 4. Consider the contract design subgame uu in which both manufacturers select U-suppliers who have private cost information.

(i) There exists a unique symmetric BNE $\left\{\left(q_{l}^{u u}, T_{l}^{u u}, q_{h}^{u u}, T_{h}^{u u}\right)\right\}$, where

$$
\begin{aligned}
q_{l}^{u u} & =\frac{A-C_{l}}{2+\gamma}+\frac{2 \gamma(1-2 \beta)\left(C_{h}-C_{l}\right)}{(2+\gamma)(2+\gamma(4 \beta-1))}, \quad T_{l}^{u u}=C_{l} q_{l}^{u u}+\left(C_{h}-C_{l}\right) q_{h}^{u u}, \\
q_{h}^{u u} & =\frac{A-C_{h}}{2+\gamma}-\frac{C_{h}-C_{l}}{2+\gamma(4 \beta-1)}, \quad T_{h}^{u u}=C_{h} q_{h}^{u u} .
\end{aligned}
$$

(ii) Each manufacturer's profit in equilibrium is $\Pi^{u u}=\frac{1}{2}\left(q_{l}^{u u}\right)^{2}+\frac{1}{2}\left(q_{h}^{u u}\right)^{2}$. Each supplier's profit is $\pi^{u u}=\frac{1}{2}\left(C_{h}-C_{l}\right) q_{h}^{u u}$.

Note first that the condition to ensure a positive order quantity from the high-cost type supplier is $A>C_{h}+\frac{(2+\gamma)\left(C_{h}-C_{l}\right)}{2+\gamma(4 \beta-1)}$. Due to information asymmetry, each manufacturer has to pay a positive information rent to the low-cost type supplier, while the high-cost supplier's profit is 0. We also observe that the information rent paid to the low-cost type increases in the market size $A$.

In the corollary below, we present the result for the subgame $c c$, which is a special case of the subgame $u$. The corollary follows from Proposition 4.

COROllary 1. When $c_{l}=c_{h}=c$, the subgame uu reduces to the subgame cc in which both manufacturers select $C$-suppliers. In this case, there is a unique symmetric Nash equilibrium $\left\{\left(q^{c c}, T^{c c}\right)\right\}$ where

$$
q^{c c}=\frac{A-c}{2+\gamma}, \quad T^{c c}=c q^{c c}
$$

Each manufacturer's profit in equilibrium is $\Pi^{c c}=\left(q^{c c}\right)^{2}$. Each supplier's profit is 0.

\subsection{Impact of Information Asymmetry}

To understand how information asymmetry affects the manufacturer competition, again we have analyzed the benchmark case where there is no information asymmetry between the suppliers and manufacturers; that is, each supplier's cost signal is known to its manufacturer but not the other manufacturer. This benchmark case boils down to the quantity competition model with cost 
uncertainty where each manufacturer does not know the competitor's cost. We relegate the detailed analysis to Appendix A, and summarize the equilibrium for this case in Lemma A.2.

We now compare Proposition 4 with Lemma A.2 to examine the impact of information asymmetry in the subgame $u$. Regarding the equilibrium quantities we obtain

$$
\begin{aligned}
q_{l}^{u u}-\bar{q}_{l}^{u u} & =\frac{\gamma(1-2 \beta)\left(C_{h}-C_{l}\right)}{(2+\gamma)(2+\gamma(4 \beta-1))} \geq 0, \\
q_{h}^{u u}-\bar{q}_{h}^{u u} & =-\frac{2(\gamma \beta+1)\left(C_{h}-C_{l}\right)}{(2+\gamma)(2+\gamma(4 \beta-1))}<0 .
\end{aligned}
$$

The above comparison reveals an interesting insight: There is an upward distortion for the low-cost type and a downward distortion for the high-cost type, which is in contrast with the screening mechanism in the single supply chain setting as well as the asymmetric competition setting in Section 4. Competition leads each manufacturer to place a larger order from the low-cost type but a smaller order from the high-cost type. The intuition is as follows: With information asymmetry, each manufacturer should order less from the high-cost supplier in order to prevent the low-cost supplier from mimicking the high-cost supplier. Under competition, each manufacturer's quantity decision depends on the expected quantity of the competitor. Due to the competitor's downward distortion of quantity when facing the high-cost type, the manufacturer's competitive advantage when facing the low-cost type supplier is exaggerated, which is reflected in the contract in which she inflates the order quantity from the low-cost type supplier.

In the following, we will explore how the equilibrium outcomes are affected by other key problem parameters. For concision, we omit the detailed results that are similar to those in Section 4. To avoid triviality we assume the market size is large enough so that both order quantities are positive in equilibrium. Specifically, we assume $A>\mu+(2 \alpha-1)(4 \gamma \beta+\gamma+6) \delta /(2-\gamma+4 \gamma \beta)$, or equivalently, $\delta<(2-\gamma+4 \gamma \beta)(A-\mu) /((2 \alpha-1)(4 \gamma \beta+\gamma+6))$.

\subsection{Effects of Cost Uncertainty and Signal Quality}

Under the subgame $u$, each manufacturer sources from a U-supplier. Similarly, we analyze the effect of cost uncertainty $\delta$ and signal accuracy $\alpha$ on each manufacturer's profit. The effects of $\delta$ and $\alpha$ on each manufacturer's profit under the subgame $u$ share the same pattern as their effects on the profit of manufacturer $j$ (the manufacturer who sources from a U-supplier) under the subgame $c u$. That is, when the market size $A$ is sufficiently large, each manufacturer's profit decreases in $\delta$ and $\alpha$. Otherwise, as $\delta$ or $\alpha$ increases, each manufacturer's profit first decreases and then increases (please refer to Appendix B for the detailed analytical results). The underlying driving forces are the same as discussed in Section 4.2 and 4.3, and hence are omitted. 


$$
\begin{array}{ccc} 
& \multicolumn{2}{c}{\text { Manufacturer } 2} \\
S_{c} & S_{u} \\
\text { Manufacturer 1 } & S_{c} & S_{u}
\end{array}\left[\begin{array}{cc}
\Pi^{c c}, \Pi^{c c} & \Pi_{1}^{c u}, \Pi_{2}^{c u} \\
\Pi_{1}^{u c}, \Pi_{2}^{u c} & \Pi^{u u}, \Pi^{u u}
\end{array}\right]
$$

Figure 1 Sourcing game with information asymmetry

\section{Sourcing Game}

Having analyzed the four subgames in the previous sections, we are ready to examine the sourcing game at the first stage. The sourcing game can be represented using the matrix in Figure 1. To avoid triviality, we assume the market size is large enough so that the order quantities for all the subgames are positive. Specifically we assume

$$
A>\max \left[c, \frac{2 c-\gamma(\mu+(2 \alpha-1) \delta)}{2-\gamma}, \frac{2 \mu+(2 \alpha-1)\left(6-\gamma^{2}\right) \delta-\gamma c}{2-\gamma}, \mu+\frac{(2 \alpha-1)(4 \gamma \beta+\gamma+6)}{2-\gamma+4 \gamma \beta} \delta\right] .
$$

To derive the equilibrium of the sourcing game, we need to compare the profits of the four subgames. Because the manufacturers are ex ante symmetric, we have $\Pi_{1}^{u c}=\Pi_{2}^{c u}$ and $\Pi_{1}^{c u}=\Pi_{2}^{u c}$ in Figure 1. Then we need to compare two pairs of profits: $\Pi^{c c}$ versus $\Pi_{2}^{c u}$, and $\Pi_{1}^{c u}$ versus $\Pi^{u u}$. Define:

$$
\Delta_{1}=\Pi^{c c}-\Pi_{2}^{c u} \quad \text { and } \quad \Delta_{2}=\Pi_{1}^{c u}-\Pi^{u u} .
$$

The signs of these differences indicate a manufacturer's preferred supplier given the competitor's sourcing strategy. For example, if $\Delta_{1}>0\left(\Delta_{2}>0\right)$, then given one manufacturer has selected a C-supplier (U-supplier), the other manufacturer will be better off choosing a C-supplier. We begin by establishing the definite relationship between $\Delta_{1}$ and $\Delta_{2}$ as shown in the following lemma.

LEMMa 1. $\Delta_{2}>\Delta_{1}$ for $\tau \in(1 / 4,1 / 2]$.

$\Delta_{2}>\Delta_{1}$ implies that a manufacturer has greater incentives to select a C-supplier given the other manufacturer has chosen a U-supplier. Alternatively, a manufacturer has greater incentives to choose a U-supplier if her competitor has chosen a C-supplier. Next, we characterize the sourcing equilibrium for the manufacturers. We start with the special cases in which the cost difference between C-supplier and U-supplier is significant (i.e., either $c \geq C_{h}$ or $c \leq \mu$ ).

Proposition 5. If $c \geq C_{h}$, the unique Nash equilibrium is $\left(S_{u}, S_{u}\right)$. If $c \leq \mu$, the unique Nash equilibrium is $\left(S_{c}, S_{c}\right)$.

Proposition 5 shows that if the high conditional cost of U-supplier is even smaller than the constant cost of C-supplier, U-supplier is more attractive in spite of its cost uncertainty, and both 
manufacturers choose U-suppliers. On the other hand, if a U-supplier does not have average cost advantage, i.e., its average cost is greater than the cost of a $\mathrm{C}$-supplier $(c \leq \mu)$, then selecting a $\mathrm{C}$-supplier is the dominant strategy, and hence the unique equilibrium is $\left(S_{c}, S_{c}\right)$.

In the following analysis, we focus on the case where $\mu<c<C_{h}$. Such a parameter setting is also consistent with the common belief that suppliers at well-established manufacturing bases (e.g., China) have higher average cost than the Southeast Asian suppliers (i.e., $\mu<c$ ). Moreover, due to less-developed infrastructure, volatile economic environment and so on, the sourcing cost from Southeast Asia conditional on a high-cost signal can be higher than that of China (i.e., $c<C_{h}$ ). We define two thresholds of $A, A_{1}$ and $A_{2}$, such that $\left.\Delta_{1}\right|_{A=A_{1}}=0,\left.\Delta_{2}\right|_{A=A_{2}}=0$, and $\Delta_{1}>0\left(\Delta_{2}>0\right)$ if and only if $A>A_{1}\left(A>A_{2}\right)$. It has been shown that $A_{1}>A_{2}$. We characterize the sourcing equilibrium in Proposition 6.

Proposition 6. Suppose $\mu<c<C_{h}$. Then the equilibria of the sourcing game with cost information asymmetry are given as follows. ${ }^{4}$

(i) If $A<A_{2}$, then $\left(S_{u}, S_{u}\right)$ is the unique Nash equilibrium;

(ii) If $A=A_{2}$, then both $\left(S_{u}, S_{u}\right)$ and $\left(S_{c}, S_{u}\right)$ are Nash equilibria;

(iii) If $A_{2}<A<A_{1}$, then $\left(S_{c}, S_{u}\right)$ is the unique Nash equilibrium;

(iv) If $A=A_{1}$, then both $\left(S_{c}, S_{u}\right)$ and $\left(S_{c}, S_{c}\right)$ are Nash equilibria;

(v) If $A>A_{1}$, then $\left(S_{c}, S_{c}\right)$ is the unique Nash equilibrium.

Proposition 6 tells that, as the market size increases the sourcing equilibrium shifts from $\left(S_{u}, S_{u}\right)$ to $\left(S_{c}, S_{u}\right)$ and further to $\left(S_{c}, S_{c}\right)$. That is, when the market size is sufficiently large, both manufacturers should use C-suppliers. This is in contrast to the intuition that as the market size becomes larger, the manufacturers should care more about the average production cost, and hence should more likely choose U-suppliers due to their lower average cost. The intuition of this result is as follows: When sourcing from U-suppliers, manufacturers need to pay information rent to the suppliers, and the information rent increases with the market size. As a result, with a larger market size, U-suppliers become less attractive while C-suppliers become more attractive, and thus the sourcing equilibrium moves from $\left(S_{u}, S_{u}\right)$ to $\left(S_{c}, S_{c}\right)$. This indicates that in an economic recession with shrinking market, manufacturers are more likely to source from U-suppliers (e.g., suppliers in Southeast Asia).

\footnotetext{
${ }^{4}$ A necessary condition for equilibrium $\left(S_{u}, S_{u}\right)$ is $A \leq A_{2}$. However, the non-negative constraint requires $A$ to be greater than a threshold, say $\underline{A}$. Therefore, $\left(S_{u}, S_{u}\right)$ is an equilibrium only when $A_{2}$ is sufficiently large; otherwise $\left(S_{u}, S_{u}\right)$ cannot be an equilibrium. Similarly, $\left(S_{c}, S_{u}\right)$ cannot be an equilibrium if $A_{1} \leq \underline{A}$.
} 


\subsection{Impact of Information Asymmetry}

In this section we examine the impact of information asymmetry on sourcing equilibrium. It is noted that there is still cost uncertainty in the symmetric information case. That is, although U-supplier's signal is known to the manufacturer sourcing from it, U-supplier's cost is still uncertain to both itself and the manufacturers. If there is no information asymmetry, we have shown in Proposition A.1 in Appendix A that the unique equilibrium is $\left(S_{u}, S_{u}\right)$ for $\mu \leq c$. The fact that U-suppliers are chosen even for $\mu=c$ means that in the absence of information asymmetry, cost uncertainty makes U-suppliers more attractive, because the manufacturers benefit from the flexibility that they can offer the contract based on the supplier's cost realization. Not surprisingly, U-supplier is more attractive and preferred if it further has a cost advantage (i.e., $\mu<c$ ).

However, in the presence of information asymmetry, the unique equilibrium is $\left(S_{c}, S_{c}\right)$ for $\mu=c$ (Proposition 5). Since if choosing a U-supplier, the manufacturers have to pay information rent to the supplier, the manufacturers' sourcing decisions involve a trade-off between the upside of cost uncertainty and the downside of information asymmetry. Our results suggest that the downside of information asymmetry for U-suppliers dominates the upside of their pure cost uncertainty.

For $\mu<c<C_{h}$, U-suppliers have (average) cost advantage as opposed to C-suppliers. In this case, the manufacturers' sourcing decisions depend on a more complicated trade-off between the cost advantage (as well as the upside of cost uncertainty) and the downside of information asymmetry. In contrast with Proposition A.1, Proposition 6 shows that, with information asymmetry, C-suppliers can be selected for sufficiently large market size $\left(A>A_{2}\right)$. We know that more information rent has to be paid to U-suppliers when the market size increases. As a consequence, selecting a Usupplier becomes unattractive to the manufacturers when the market size is large enough. This suggests that the downside of information rent can offset the overall benefit from the U-suppliers' nominal cost advantage and cost uncertainty. These results highlight the importance of considering information asymmetry for the manufacturers when designing their sourcing strategies.

Next, we investigate how different problem parameters affect the outcome of the sourcing game under information asymmetry by investigating the sensitivity of $A_{1}$ and $A_{2}$ to those parameters. By Proposition 6, increasing (decreasing) $A_{1}$ and/or $A_{2}$ implies that U-suppliers (C-suppliers) will more likely be chosen in the equilibrium.

\subsection{Effect of Cost Uncertainty}

First, we consider the effect of cost uncertainty and present the result in the following proposition.

Proposition 7. Suppose $\mu<c<C_{h}$. Then there exist two thresholds $\tilde{\delta}, \hat{\delta} \in((c-\mu) /(2 \alpha-$ 1), $2(c-\mu) /(2 \alpha-1)]$ with $\tilde{\delta} \leq \hat{\delta}$, such that:

(i) Both $A_{1}$ and $A_{2}$ decrease in $\delta$ for $\delta \leq \tilde{\delta}$; 
(ii) $A_{1}$ increases but $A_{2}$ decreases in $\delta$ for $\delta \in[\tilde{\delta}, \hat{\delta}]$;

(iii) Both $A_{1}$ and $A_{2}$ increase in $\delta$ for $\delta \geq \hat{\delta}$.

Proposition 7 shows that increasing cost uncertainty of the U-supplier may influence the results in multiple directions. When the cost uncertainty is small $(\delta \leq \tilde{\delta})$, U-suppliers become more attractive for even smaller uncertainty. When the cost uncertainty is intermediate $(\tilde{\delta} \leq \delta \leq \hat{\delta})$, increasing uncertainty leads the manufacturers to more likely use the diversified strategy in equilibrium. When the cost uncertainty is already large $(\delta \geq \hat{\delta})$, increasing uncertainty drives up the values of $A_{1}$ and $A_{2}$, and hence U-suppliers become more attractive for even larger uncertainty. Why does increasing cost uncertainty make U-suppliers more attractive when the initial uncertainty is large? This is because by Proposition 2 and Proposition B.1 (in Appendix B), if the current uncertainty is large, under both $c u$ and $u u$ the manufacturer who selects a U-supplier will benefit from increased cost uncertainty due to the dominance of the positive effect of more variable cost over the negative effect of greater distortion driven by information asymmetry.

In contrast, when the current uncertainty is small, increasing cost uncertainty hurts the manufacturer who chooses a U-supplier and benefits the manufacturer who chooses a C-supplier (Propositions 2 and B.1). Therefore, as cost uncertainty increases, under $c u$ the manufacturer who selects a U-supplier tends to change to a $\mathrm{C}$-supplier, and under $u$ one of the manufacturers may break the equilibrium by deviating to a C-supplier.

When the uncertainty is in the intermediate range, reducing the uncertainty further will make the symmetric sourcing equilibrium more likely happen. This is because as $\delta$ increases, the intermediate values of $\delta$ are sufficiently large to make U-suppliers more likely be chosen if the starting structure is $\left(S_{c}, S_{c}\right)$, but if the starting structure is $\left(S_{u}, S_{u}\right)$ the $\delta$ values are not large enough and hence C-suppliers become more likely selected.

Cost uncertainty captured by $\delta$ measures the extent to which U-supplier's cost is estimated to deviate from the average cost. This is mainly from the information standpoint. Reducing cost uncertainty represents an effort to obtain a better cost estimate but not necessarily to reduce the average cost. For example, the firms could consult industry experts or conduct more research about the business environment and manufacturing process of the suppliers. Recent advances in technologies such as big data may help the firms to collect a large amount of data necessary for obtaining a better cost estimate. Government could also play an important role in terms of revealing more information to the outside so that the outsiders can better estimate potential costs. For example, one of the goals of The ASEAN Good Regulatory Practice Core Principles adopted 
at the 50th ASEAN Economic Ministers' Meeting is to ensure that "access to accurate, easy-tounderstand, and accessible information on the regulations should be made available for relevant stakeholders". ${ }^{5}$

The uncertainties in Southeast Asian suppliers might be gradually reduced considering the governments' effort in these countries. Our study implies that over the period with large cost uncertainty, decreasing the uncertainty actually makes U-suppliers less likely be chosen by the manufacturers in equilibrium. Only when the cost uncertainty is already not too large can reducing the uncertainty further lead the U-suppliers to be more likely chosen.

\section{3. $\quad$ Effect of Signal Quality}

The effect of signal quality on the sourcing equilibrium is summarized below. Define $N=20-4 \gamma-$ $8 \gamma^{2}+\gamma^{4}$ and $M=20-8 \gamma^{2}+\gamma^{4}$.

Proposition 8. Suppose $\mu<c<C_{h}$ and $\tau=1 / 4$. Then there exist two thresholds $\tilde{\alpha}$ and $\hat{\alpha}$ $(\tilde{\alpha}<\hat{\alpha})$ such that:

(i) If $(c-\mu) / \delta<\frac{N-\sqrt{N^{2}-4(1-\gamma) N}}{4(1-\gamma)}$, then both $A_{1}$ and $A_{2}$ decrease in $\alpha$ for $\alpha<\tilde{\alpha}$, $A_{1}$ increases but $A_{2}$ decreases in $\alpha$ for $\alpha \in(\tilde{\alpha}, \hat{\alpha})$, and both $A_{1}$ and $A_{2}$ increase in $\alpha$ for $\alpha>\hat{\alpha}$;

(ii) If $(c-\mu) / \delta \in\left(\frac{N-\sqrt{N^{2}-4(1-\gamma) N}}{4(1-\gamma)}, \frac{M-\sqrt{M^{2}-4 M}}{4}\right)$, then both $A_{1}$ and $A_{2}$ decrease in $\alpha$ for $\alpha<\tilde{\alpha}$, and $A_{1}$ increases but $A_{2}$ decreases in $\alpha$ for $\alpha>\tilde{\alpha}$;

(iii) If $(c-\mu) / \delta>\frac{M-\sqrt{M^{2}-4 M}}{4}$, then both $A_{1}$ and $A_{2}$ decrease in $\alpha$.

As a note, the sensitivity result about $A_{1}$ does not require $\tau=1 / 4$ (i.e., independent costs for U-suppliers). Although the result about $A_{2}$ is derived for the case of $\tau=1 / 4$, we have verified by conducting extensive numerical experiments that the above results also hold for general cases.

The proposition implies increasing signal accuracy can make U-suppliers more likely chosen and C-suppliers less likely chosen (i.e., $A_{1}, A_{2}$ increases) only when both signal accuracy $\alpha$ and cost uncertainty $\delta$ are above a certain level. This is because a more accurate signal for the supplier makes the manufacturer at a severer cost disadvantage, but on the other hand allows the manufacturer to design a more targeted contract menu. For larger cost uncertainty, the marginal benefit from a more targeted contract menu is larger. For already high signal accuracy, the marginal detrimental effect of asymmetric information is limited, and hence can be dominated by the large benefit from a more targeted contract menu when cost uncertainty is high.

\footnotetext{
${ }^{5}$ https://asean.org/storage/2017/11/ASEAN-GRP-Core-Principles-FINAL-ENDORSED.pdf
} 


\subsection{Effect of Competition Intensity}

To study the effect of competition intensity on the manufacturers' sourcing strategies, we examine how $A_{1}$ and $A_{2}$ change in the value of $\gamma$.

Proposition 9. Suppose $\mu<c<C_{h}$.

(i) If $\delta /(c-\mu) \geq 1 /((1-\sqrt{3} / 2)(2 \alpha-1)), A_{1}$ increases in $\gamma$; otherwise, $A_{1}$ first increases and then decreases in $\gamma$.

(ii) If $\tau \geq \frac{1}{4}+\frac{1}{8(2 \alpha-1)^{2}}$ or $\delta /(c-\mu) \geq 1 /\left((2 \alpha-1)\left(1-2 \sqrt{1-2(4 \tau-1)(2 \alpha-1)^{2}}\right)\right), A_{2}$ decreases in $\gamma$; otherwise, $A_{2}$ first increases and then decreases in $\gamma$.

Proposition 9 shows that when the cost uncertainty $\delta$ (equivalently, $\delta /(c-\mu)$ ) is sufficiently large, intensified competition drives the manufacturers to diversify their sourcing strategy (i.e., $A_{1}$ increases or $A_{2}$ decreases). This is because for sufficiently large cost uncertainty, the impact of more intense competition on the manufacturers' profits under $\left(S_{c}, S_{u}\right)$ is not as strong as that under $\left(S_{c}, S_{c}\right)$ and $\left(S_{u}, S_{u}\right)$.

However, for not sufficiently large cost uncertainty, the impact of intensified competition on the sourcing equilibrium can be non-monotone, depending on the cost correlation of U-suppliers, $\tau$, and the current competition intensity. For not very large $\delta$, the profit of the manufacturer sourcing from U-supplier under $c u$ decreases in $\gamma$ more significantly than the manufacturers under $\left(S_{c}, S_{c}\right)$ when $\gamma$ is large, driving $\left(S_{c}, S_{c}\right)$ to be more likely the equilibrium for large $\gamma$.

When the correlation of U-suppliers' cost $\tau$ and cost uncertainty $\delta$ are small, for the range of moderate competition intensity, increasing competition intensity has weaker negative impact on the manufacturers' profits under $u u$ and hence $\left(S_{u}, S_{u}\right)$ is more likely the equilibrium. However, for the range of high competition intensity, the negative impact of intensified competition is stronger under the symmetric structure $u u$, and hence $\left(S_{c}, S_{u}\right)$ tends to be the equilibrium instead of $\left(S_{u}, S_{u}\right)$.

\subsection{Effect of Increasing Cost of C-Suppliers}

Not surprisingly, the increasing cost of C-suppliers will drive more manufacturers to source from U-suppliers (both $A_{1}$ and $A_{2}$ increase in $c$ ). This reflects the reality that due to fast-rising wages in China, many manufacturers choose to source from suppliers in Southeast Asia. How will this change affect the manufacturers' profitability? Next we shed some light on this question. For analytical tractability, in the following we consider the case of $\gamma=1$. Define

$$
\begin{aligned}
& \underline{\kappa}=\sqrt{(A-c)^{2}-9(2 \alpha-1)^{2} \delta^{2}}-(A+c-2 \mu-2(2 \alpha-1) \delta), \\
& \bar{\kappa}=\min \left[\frac{\mu+(2 \alpha-1) \delta-c}{2}, \frac{A+\mu+(2 \alpha-1) \delta-2 c}{2}-\sqrt{\frac{(A-\mu-(2 \alpha-1) \delta)^{2}}{4}+\frac{9(2 \alpha-1)^{2} \delta^{2}}{(1+4 \beta)^{2}}}\right] .
\end{aligned}
$$


Proposition 10. Suppose $\mu<c<C_{h}$ and $A>A_{1}$. Then an increase of $c$ by $\kappa>0$, where $\underline{\kappa}<\kappa<\bar{\kappa}$, shifts the sourcing equilibrium from $\left(S_{c}, S_{c}\right)$ to $\left(S_{c}, S_{u}\right)$ and makes both manufacturers better off.

To see an example, consider $A=35, \mu=10, \delta=5, c=12, \alpha=1, \tau=0.4$, and thus $\beta=0.4$. Under these parameters, we obtain $A_{1}=33.75$, so $A>A_{1}$ and $\left(S_{c}, S_{c}\right)$ is the equilibrium, under which both manufacturers' profits are 58.78. Now suppose $c$ increases by $10 \%$, or 1.2 . Then it can be shown that the sourcing equilibrium becomes $\left(S_{c}, S_{u}\right)$, under which the manufacturer that remains sourcing from $S_{c}$ earns a profit of 61.88 , and the manufacturer that switches to sourcing from $S_{u}$ earns 61.80, both greater than 58.78. In fact, the results in Proposition 10 carry over to more general cases where $\gamma<1$. For example, for $\gamma=0.95$ and all the rest of the parameter values the same as above, we obtain $A_{1}=33.89$, so $\left(S_{c}, S_{c}\right)$ is still the equilibrium and each manufacturer makes a profit of 60.79 . After the $10 \%$ increase in $c$, we can show that $A=35$ is between the two new thresholds of $A$, so the equilibrium becomes $\left(S_{c}, S_{u}\right)$. Meanwhile, the manufacturer who still chooses a C-supplier makes a profit of 61.10 and the one who chooses a U-supplier makes a profit of 63.78. Again, both manufacturers are better off.

The above result comes from the fact that an appropriate increase in $c$ moves the equilibrium from $\left(S_{c}, S_{c}\right)$ to $\left(S_{c}, S_{u}\right)$. With an increase in $c$, C-suppliers become less attractive, and the manufacturer who switches to a U-supplier enjoys more average cost advantage. This benefits the switching manufacturer but hurts the non-switching one. However, the switching manufacturer also needs to pay information rent to the chosen U-supplier, which hurts the switching manufacturer but benefits the non-switching one. The overall effects on the two manufacturers' profits depend on which of the two counteractive forces dominates. The condition $\kappa>\underline{\kappa}$ is to guarantee that the cost increase is sufficiently large so that $\left(S_{c}, S_{c}\right)$ is no longer the equilibrium. The condition $\kappa<\bar{\kappa}$ is to guarantee that the cost increase is not too large so that only one manufacturer switches from $S_{c}$ to $S_{u}$. Meanwhile, $\kappa>\underline{\kappa}$ ensures that the manufacturer that switches to $S_{u}$ is better off and $\kappa<\bar{\kappa}$ ensures that the manufacturer that remains to source from $S_{c}$ is also better off.

It is shown that $\underline{\kappa}<\bar{\kappa}$ in Proposition 10 does not hold if $c<\mu$. This means in order for both manufacturers to be better off due to one manufacturer switching to $S_{u}$, the average cost of Usuppliers has to be lower than the constant cost of C-suppliers. This condition matches the situation that Chinese suppliers are generally believed to have higher costs than Southeast Asian suppliers. It is also worth noting that the result in Proposition 10 does not occur under symmetric information. Proposition A.1 shows that, in our focal case where $\mu<c,\left(S_{u}, S_{u}\right)$ is the Nash equilibrium. Since an increase in $c$ can only make C-suppliers less attractive and U-suppliers more attractive, neither manufacturer will switch and thus $\left(S_{u}, S_{u}\right)$ remains the Nash equilibrium. Finally, it is obvious that 
the above result does not occur when there is no competition between the manufacturers. Overall, the above discussion highlights the joint impact of cost difference, asymmetric information, and competition on the manufacturers' sourcing strategies and profits.

\section{Conclusion}

The wages of Chinese workers have seen a significant increase over the past years, and many firms have started to source from Southeast Asian countries. However, an important caveat is that firms may have less knowledge about these suppliers' cost structures, especially when trading with them for the first time; the informational disadvantage and increased uncertainty may undermine the benefit from lower costs. This paper examines the trade-off between cost advantage and cost information asymmetry/uncertainty in a sourcing game where competing firms choose between a C-supplier with transparent, certain cost (e.g., Chinese supplier) and a U-supplier with nontransparent, uncertain cost (e.g., Southeast Asian supplier). Our focus is on understanding the nuances and subtleties of firms' sourcing strategies considering both information uncertainty and asymmetry.

We characterize the equilibrium outcome for the firms and conduct an extensive sensitivity analysis to examine how the equilibrium outcome is affected by various parameters, including the market size, cost uncertainty, accuracy of the U-supplier's private signal, and competition intensity. We show that, as the market size increases, firms are more likely to choose C-suppliers despite their higher average cost. Reducing cost uncertainty of the U-suppliers neither implies that the firms will choose them more often, nor does it bring more profits to the firms. This cautions that sourcing firms need to be careful when conducting any cost uncertainty reduction activities. The U-suppliers' more accurate private information about their own costs can benefit them in terms of the chance of being selected only when both information accuracy and cost uncertainty are above a certain level. Our results also show that if the cost uncertainty is sufficiently large, increasing competition intensity drives firms to diversify their sourcing strategy; otherwise, it can favor either C-suppliers or U-suppliers in terms of the chance of being selected. Furthermore, an increase in the C-supplier's cost may lead to a win-win situation for both firms. The above insights provide useful guidance for supply chain professionals when making their sourcing decisions.

On the theoretical side, we also identify an interesting ordering behavior in such a competitive setting with information asymmetry. Under the sourcing structure $c u$, we find that the firms' quantity distortion compared to the symmetric information setting presents contrasting patterns with the standard result "no distortion at the top but downward distortion at the bottom" that occurs in a single firm's optimal mechanism.

This research opens several avenues for future research. First, it has been considered that the firms engage in quantity competition in this paper. It would be interesting to study how results 
will change if one considers price competition. Second, in this paper the firms offer a take-it-orleave-it contract to $\mathrm{C}$-suppliers and a menu of contracts to U-suppliers. Instead, considering a Nash bargaining framework, especially with information asymmetry, will be a promising research direction. Third, when sourcing from suppliers in a new region, there are many factors (e.g., quality, delivery performance, social responsibility) for firms to consider besides cost. How those factors may affect firms' sourcing decisions also deserves more research attention.

\section{References}

Bolandifar E, Feng T, Zhang F (2018) Simple contracts to assure supply: Noncontracible capacity and asymmetric cost information. Manufacturing \& Service Operations Management 20(2): 217-231.

Burnetas A, Gilbert SM, Smith CE (2007) Quantity discounts in single-period supply contracts with asymmetric demand information. IIE Transactions 39(5): 465-479.

Cachon GP, Harker PT (2002) Competition and outsourcing with scale economies. Management Science 48(10): 1314-1333.

Cachon GP, Zhang F (2006) Procuring fast delivery: Sole-sourcing with information asymmetry. Management Science 52(6): 881-896.

Cachon GP, Lariviere M (2001) Contracting to assure supply: How to share demand forecasts in a supply chain. Management Science 47(5): 629-646.

Chan D (2014) Manufacturing beyond China. Forbes. August 25.

Choi SC (1996) Price competition in a duopoly common retailer channel. Journal of Retailing 72(2):117-134.

Chu K (2013) China manufacturers survive by moving to Asian neighbors. Wall Street Journal. May 1.

Corbett CJ (2001) Stochastic inventory systems in a supply chain with asymmetric information: Cycle stocks, safety stocks, and consignment stock. Operations Research 49(4): 487-500.

Corbett CJ, de Groote X (2000) A supplier's optimal quantity discount policy under asymmetric information. Management Science 46(3): 444-450.

Corbett CJ, Zhou D, Tang CS (2004) Designing supply contracts: Contract type and information asymmetry. Management Science 50(4): 550-559.

Costello AM (2013) Mitigating incentive conflicts in inter-firm relationships: Evidence from long-term supply contracts. Journal of Accounting and Economics 56: 19-39.

Dasgupta P, Hammond P, Maskin E (1979) The implementation of social choice rules: Some results on incentive compatibility. Review of Economic Studies 46: 185-216.

Dipak T (2002) 9 Reasons to switch to a single supplier system. Quality Progress 35(3): 61-64.

Epstein LG, Peters M (1999) A revelation principle for competing mechanisms. Journal of Economic Theory 88: $119-160$.

Etro F, Cella M (2013) Equilibrium principal-agent contracts: Competition and R\&D incentives. Journal of Economics \& Management Strategy 22(3): 488-512. 
Feng Q, Lu LX (2012) The strategic perils of low cost outsourcing. Management Science 58(6):1196-1210.

Fershtman C, Judd KL (1987) Equilibrium incentives in oligopoly. The American Economic Review 77(5):927-940.

Gilbert SM, Xia Y, Yu G (2006) Strategic outsourcing for competing OEMs that face cost reduction opportunities. IIE Transactions 38(11): 903-915.

Ha AY (2001) Supplier-buyer contracting: Asymmetric cost Information and the cut-off level policy for buyer participation. Naval Research Logistics 48(1): 41-64.

Ha AY, Tong S (2008) Contracting and information sharing under supply chain competition. Management Science 54(4): 701-715.

Ha AY, Tong S, Zhang H (2011) Sharing demand information in competing supply chains with production diseconomies. Management Science 57(3): 566-581.

Hortacsu A, Martinez-Jerez FA, Douglas J (2009) The geography of trade in online transactions: Evidence from eBay and MercadoLibre. American Economic Journal: Microeconomics 1(1): 53-74.

Hu B, Qi A (2018) Optimal procurement mechanisms for assembly. Manufacturing 83 Service Operations Management, 20(4): 655-666.

Kayis E, Erhun F, Plambeck EL (2013) Delegation vs. control of component procurement under asymmetric cost information and simple contracts. Manufacturing $\&$ Service Operations Management 15(1): 45-56.

Kostamis I, Duenyas I (2011) Purchasing under asymmetric demand and cost information: When is more private information better? Operations Research 59(4): 914-928.

Li Z, Gilbert S, Lai G (2015a) Supplier encroachment as an enhancement or a hindrance to nonlinear pricing. Production and Operations Management 24(1): 89-109.

Li Z, Ryan J, Shao L, Sun D (2015b) Supply contract design for competing heterogeneous suppliers under asymmetric information. Production and Operations Management 24(5): 791-807.

Lutze H, Özer Ö (2008) Promised lead-time contracts under asymmetric information. Operations Research 56(4): 898-915.

McAfee RP (1999) Mechanism design by competing sellers. Econometrica 61(6): 1281-1312.

McGuire TW, Staelin R (1983) An industry equilibrium analysis of downstream vertical integration. Marketing Science 2(2): 161-191.

Moorthy KS (1998) Strategic decentralization in channels. Marketing Science 7(4): 335-355.

Myerson R (1979) Incentive-compatibility and the bargaining problem. Econometrica 47: 61-73.

Oh S, Özer Ö (2013) Mechanism design for capacity planning under dynamic evolutions of asymmetric demand forecasts. Management Science 59(4): 987-1007.

Özer Ö, Raz G (2011) Supply chain sourcing under asymmetric information. Production and Operations Management 20(1): 92-115.

Özer Ö, Wei W (2006) Strategic commitments for an optimal capacity decision under asymmetric forecast information. Management Science 52(8): 1238-1257. 
Peters M, Severinov S (1997) Competition among sellers who offer auctions instead of prices. Journal of Economic Theory 75: 141-179.

PwC (2010) Why global sourcing? Why now? Creating competitive advantage in today's volatile marketplace. December 2010.

Sirkin HL, Zinser M, Rose J (2014) The shifting economics of global manufacturing. The Boston Consulting Group. August 19.

Swinney R, Netessine S (2009) Long-term contracts under the threat of supplier default. Management $\mathscr{E}$ Service Operations Management 11(1): 109-127.

The Economist (2015) The future of factory Asia: A tightening grip. May 14.

Vives X (1999) Oligopoly Pricing, MIT Press, Cambridge, MA.

Woetzel J, Tonby O, Thompson F, Burtt P, Lee G (2014) Southeast Asia at the crossroads: Three paths to prosperity, McKinsey Global Institute.

Wu X, Zhang F (2014) Home or overseas? An analysis of sourcing strategies under competition. Management Science 60(5): 1223-1240.

Yang Z, Aydin G, Babich V, Beil D (2009) Supply disruptions, asymmetric information and a backup production option. Management Science 55(2): 192-209.

Zhang F (2010) Procurement mechanism design in a two-echelon inventory system with price-sensitive demand. Manufacturing \& Service Operations Management 12(4): 608-626. 


\section{Online Appendices to "Sourcing Competition under Cost Uncertainty and Information Asymmetry"}

\section{Appendix A: Symmetric Information Benchmark}

To investigate the role of information asymmetry, this appendix analyzes an auxiliary benchmark case in which there is no information asymmetry between manufacturers and suppliers. Similar to the analysis for the case with information asymmetry, we first examine the four subgames and then characterize the sourcing equilibrium for the manufacturers.

\section{A.1. Contract Design Subgame $c u(u c)$}

Because of the symmetry of subgame $c u$ and subgame $u c$, we focus on subgame $c u$ without loss of generality. In this benchmark, no information asymmetry exists between manufacturer $j$ and supplier $j$, but supplier $j$ 's cost signal is still unknown to manufacturer $i$. In terms of the model formulation, the only difference from the case with information asymmetry is that, for manufacturer $j$ 's best response problem, the IC constraints are not required. Following the same approach as for the case with information asymmetry, we characterize the equilibrium for the case without information asymmetry in Lemma A.1.

Lemma A.1. Consider the contract design subgame cu in which manufacturer $i$ selects a C-supplier and manufacturer $j$ selects a U-supplier who has no private cost information. (i) There exists a unique BNE $\left\{\left(\bar{q}_{i}^{c u}, \bar{T}_{i}^{c u}\right),\left(\bar{q}_{j l}^{c u}, \bar{T}_{j l}^{c u}, \bar{q}_{j h}^{c u}, \bar{T}_{j h}^{c u}\right)\right\}$, where

$$
\begin{aligned}
& \bar{q}_{i}^{c u}=\frac{A-c}{2+\gamma}+\frac{\gamma\left(C_{l}+C_{h}-2 c\right)}{2\left(4-\gamma^{2}\right)}, \quad \bar{T}_{i}^{c u}=c \bar{q}_{i}^{c u}, \\
& \bar{q}_{j l}^{c u}=\frac{A-C_{l}}{2+\gamma}+\frac{\gamma^{2}\left(C_{l}-C_{h}\right)-4 \gamma\left(C_{l}-c\right)}{4\left(4-\gamma^{2}\right)}, \quad \bar{T}_{j l}^{c u}=C_{l} \bar{q}_{j l}^{c u}, \\
& \bar{q}_{j h}^{c u}=\frac{A-C_{h}}{2+\gamma}+\frac{\gamma^{2}\left(C_{h}-C_{l}\right)-4 \gamma\left(C_{h}-c\right)}{4\left(4-\gamma^{2}\right)}, \quad \bar{T}_{j h}^{c u}=C_{h} \bar{q}_{j h}^{c u} .
\end{aligned}
$$

(ii) The manufacturers' profits in equilibrium are $\bar{\Pi}_{i}^{c u}=\left(\bar{q}_{i}^{c u}\right)^{2}$ and $\bar{\Pi}_{j}^{c u}=\frac{1}{2}\left(\bar{q}_{j l}^{c u}\right)^{2}+\frac{1}{2}\left(\bar{q}_{j h}^{c u}\right)^{2}$. Each supplier's profit is 0 .

From Lemma A.1 we show that the expected quantity of manufacturer $j$ is $E\left[\bar{q}_{j}^{c u}\right]=\frac{2(A-\mu)-\gamma(A-c)}{4-\gamma^{2}}$, where $\mu=\left(c_{l}+c_{h}\right) / 2$. For convenience, let $c_{h}=\mu+\delta$ and $c_{l}=\mu-\delta$. We can rewrite the equilibrium quantities and profits given in Lemma A.1. Using the results of Lemma A.1, we explore how cost uncertainty, measured by $\delta$, affects the competition between the manufacturers. Regarding order quantities, it is straightforward to show that $\bar{q}_{i}^{c u}$ is independent of $\delta, \bar{q}_{j l}^{c u}$ increases in $\delta$, and $\bar{q}_{j h}^{c u}$ decreases in $\delta$. For equilibrium profits, we observe that $\bar{\Pi}_{i}^{c u}$ is independent of $\delta$, so cost uncertainty does not affect the profit of the manufacturer who chooses a C-supplier. For manufacturer $j$ we obtain $\partial \bar{\Pi}_{j}^{c u} / \partial \delta=(2 \alpha-1)^{2} \delta / 2>0$, so the profit of the manufacturer who chooses a U-supplier increases in $\delta$. This implies that, in subgame $c u$ with symmetric information, the manufacturer who chooses a U-supplier benefits from larger cost uncertainty. 


\section{A.2. Contract Design Subgame $u u(c c)$}

Subgame $c c$ under symmetric information is the same as that under asymmetric information. Thus, Corollary 1 also applies to the case with symmetric information. In the benchmark for subgame $u$, the suppliers have no private information about their cost signals. The manufacturers can design a contract such that the suppliers receive their reservation profits only. To derive the equilibrium we first examine each manufacturer's best response. Given manufacturer $j$ 's contract $\left(q_{j l}, T_{j l}, q_{j h}, T_{j h}\right)$, we formulate manufacturer $i$ 's best response problem as follows:

$$
\begin{aligned}
\max _{q_{i l}, T_{i l}, q_{i h}, T_{i h} \geq 0} \Pi_{i}= & \frac{1}{2}\left[\left(A-q_{i l}-\gamma\left(2 \beta q_{j l}+(1-2 \beta) q_{j h}\right)\right) q_{i l}-T_{i l}\right] \\
& +\frac{1}{2}\left[\left(A-q_{i h}-\gamma\left((1-2 \beta) q_{j l}+2 \beta q_{j h}\right)\right) q_{i h}-T_{i h}\right],
\end{aligned}
$$

subject to the participation constraints $T_{i l}-C_{l} q_{i l} \geq 0$ and $T_{i h}-C_{h} q_{i h} \geq 0$. Solving the above best response problem for both manufacturers, we obtain the equilibrium in Lemma A.2.

Lemma A.2. Consider the contract design subgame uu in which both manufacturers select U-suppliers who have no private cost information. (i) There exists a unique symmetric $B N E\left\{\left(\bar{q}_{l}^{u u}, \bar{T}_{l}^{u u}, \bar{q}_{h}^{u u}, \bar{T}_{h}^{u u}\right)\right\}$, where

$$
\begin{array}{ll}
\bar{q}_{l}^{u u}=\frac{A-C_{l}}{2+\gamma}+\frac{\gamma(1-2 \beta)\left(C_{h}-C_{l}\right)}{(2+\gamma)(2+\gamma(4 \beta-1))}, & \bar{T}_{l}^{u u}=C_{l} \bar{q}_{l}^{u u}, \\
\bar{q}_{h}^{u u}=\frac{A-C_{h}}{2+\gamma}-\frac{\gamma(1-2 \beta)\left(C_{h}-C_{l}\right)}{(2+\gamma)(2+\gamma(4 \beta-1))}, & \bar{T}_{h}^{u u}=C_{h} \bar{q}_{h}^{u u} .
\end{array}
$$

(ii) Each manufacturer's profit in equilibrium is $\bar{\Pi}^{u u}=\frac{1}{2}\left(\bar{q}_{l}^{u u}\right)^{2}+\frac{1}{2}\left(\bar{q}_{h}^{u u}\right)^{2}$. Each supplier's profit is 0 .

From Lemma A.2, we find that $E\left[\bar{q}^{u u}\right]=\frac{A-\mu}{2+\gamma}$, where $\mu=\left(c_{l}+c_{h}\right) / 2$. We are interested in the effect of cost uncertainty, represented by $\delta=\left(c_{l}+c_{h}\right) / 2$, on the competition between the manufacturers. We show that $\partial \bar{\Pi}^{u u} / \partial \delta=2(2 \alpha-1)^{2} \delta /\left((2+(4 \beta-1) \gamma)^{2}\right)>0$, so the manufacturers' profits increase in $\delta$. This implies that increasing cost uncertainty benefits both manufacturers in the absence of information asymmetry.

\section{A.3. Sourcing Game}

After analyzing the four subgames, we are now ready to characterize the sourcing equilibrium in the first stage. We use a matrix in Figure A.1 to represent the supplier selection game. By symmetry, we have $\bar{\Pi}_{1}^{u c}=\bar{\Pi}_{2}^{c u}$ and $\bar{\Pi}_{1}^{c u}=\bar{\Pi}_{2}^{u c}$. For this benchmark, we assume $A>$

Manufacturer 2

$$
\begin{array}{ccc}
S_{c} & S_{u} \\
\text { Manufacturer 1 } & S_{c} & S_{u}
\end{array}\left[\begin{array}{cc}
\Pi^{c c}, \Pi^{c c} & \bar{\Pi}_{1}^{c u}, \bar{\Pi}_{2}^{c u} \\
\bar{\Pi}_{1}^{u c}, \bar{\Pi}_{2}^{u c} & \bar{\Pi}^{u u}, \bar{\Pi}^{u u}
\end{array}\right]
$$

\section{Figure A.1 Sourcing game with symmetric information}

$\max \left[c,\left(4 \mu+(2 \alpha-1)\left(4-\gamma^{2}\right) \delta-2 \gamma c\right) /(4-2 \gamma),(2 c-\gamma \mu) /(2-\gamma), \mu+(2 \alpha-1)(2+\gamma) \delta /(2-\gamma+4 \gamma \beta)\right] \quad$ such that all the quantities are positive. Consistent with the observation that Chinese suppliers generally have higher average costs than their Southeast Asian counterparts, we focus on the case with $\mu \leq c$. We summarize the sourcing equilibrium for the symmetric information setting in Proposition A.1. 
Proposition A.1. If $\mu \leq c$, then the unique Nash equilibrium is $\left(S_{u}, S_{u}\right)$.

Proposition A.1 shows that, if the average cost of a U-supplier is no greater than the constant cost of a C-supplier, then the unique sourcing equilibrium is that both manufacturers choose U-suppliers. This is because cost uncertainty benefits both manufacturers in a way we show in subgame $u u$. As a result, sourcing from a U-supplier is a dominant strategy for the manufacturers.

\section{Appendix B: Detailed Results under Subgame $u u$}

\section{B.1. Effect of Cost Uncertainty $\delta$}

We summarize the effect of cost uncertainty on each player's profit in Proposition B.1. Define

$$
\delta_{m}^{u u}=\frac{(A-\mu)((4 \beta-1) \gamma+2)^{2}}{(2 \alpha-1)\left(\left(16 \beta^{2}-8 \beta+5\right) \gamma^{2}+4(4 \beta+3) \gamma+20\right)} .
$$

Proposition B.1. Under the subgame uu where the U-suppliers have private cost information, $\frac{\partial \Pi^{u u}}{\partial \delta}<0$ for $\delta<\delta_{m}^{u u}$, and $\frac{\partial \Pi^{u u}}{\partial \delta}>0$ for $\delta>\delta_{m}^{u u}$.

\section{B.2. Effect of Signal Accuracy $\alpha$}

The analysis for the effect of $\alpha$ on the manufacturers' profits is much involved and intractable. Therefore, we analytically focus on the case with independent costs between U-suppliers (i.e., $\tau=1 / 4$ ), and resort to numerical analysis for general cases. Each manufacturer's equilibrium profit is $\Pi^{u u}=\frac{1}{2}\left(q_{l}^{u u}\right)^{2}+\frac{1}{2}\left(q_{h}^{u u}\right)^{2}$. Define $\alpha_{m}^{u u}=\frac{A+\left(\gamma^{2}+4 \gamma+5\right) \delta-\mu}{2\left(\gamma^{2}+4 \gamma+5\right) \delta}$.

Proposition B.2. Under the subgame uu where the U-suppliers have private independent cost information (i.e., $\tau=1 / 4$ ), $\frac{\partial \Pi^{u u}}{\partial \alpha}<0$ for $\alpha<\alpha_{m}^{u u}$ and $\frac{\partial \Pi^{u u}}{\partial \alpha}>0$ for $\alpha>\alpha_{m}^{u u}$.

\section{Appendix C: Extension to Multi-Point Distributions}

In the paper, we assume U-supplier's cost follows a two-point distribution. Here we show how we extend to a three-point distribution by still using the parameter $\delta$ to capture cost uncertainty and $\alpha$ to capture signal accuracy, and then show how to extend to a general multi-point distribution.

Three-point distribution: Suppose U-supplier's cost follows a three-point distribution with $\operatorname{Pr}\left(\hat{c}=c_{l}\right)=$ $\operatorname{Pr}\left(\hat{c}=c_{m}\right)=\operatorname{Pr}\left(\hat{c}=c_{h}\right)=1 / 3$ where $c_{m}=\left(c_{l}+c_{h}\right) / 2$ and $c_{l}<c_{h}$. These three cost levels can be represented using two parameters $\mu$ and $\delta$; that is, $c_{l}=\mu-\delta, c_{m}=\mu$ and $c_{h}=\mu+\delta$. The range of $\hat{c}$ is $2 \delta$ and the standard deviation of $\hat{c}$ is $\frac{\sqrt{6}}{3} \delta$. Thus, we can use $\delta$ to measure the extent of cost uncertainty.

Suppose the probabilities of signals conditional on costs are given by

$$
\begin{aligned}
\operatorname{Pr}\left(s_{l} \mid c_{l}\right)=\operatorname{Pr}\left(s_{h} \mid c_{h}\right) & =\alpha, \\
\operatorname{Pr}\left(s_{l} \mid c_{m}\right)=\operatorname{Pr}\left(s_{h} \mid c_{m}\right) & =\frac{1}{2}, \\
\operatorname{Pr}\left(s_{l} \mid c_{h}\right)=\operatorname{Pr}\left(s_{h} \mid c_{l}\right) & =1-\alpha,
\end{aligned}
$$

where $\alpha \in(1 / 2,1]$ measures the signal quality or accuracy.

Using Bayes' Theorem, we can calculate the posterior probabilities based on the above prior and conditional probabilities. We have

$$
\begin{aligned}
\operatorname{Pr}\left(c_{l} \mid s_{l}\right) & =\frac{\operatorname{Pr}\left(s_{l} \mid c_{l}\right) \operatorname{Pr}\left(c_{l}\right)}{\operatorname{Pr}\left(s_{l} \mid c_{l}\right) \operatorname{Pr}\left(c_{l}\right)+\operatorname{Pr}\left(s_{l} \mid c_{m}\right) \operatorname{Pr}\left(c_{m}\right)+\operatorname{Pr}\left(s_{l} \mid c_{h}\right) \operatorname{Pr}\left(c_{h}\right)} \\
& =\frac{\alpha \cdot 1 / 3}{\alpha \cdot 1 / 3+1 / 2 \cdot 1 / 3+(1-\alpha) \cdot 1 / 3}=\frac{2}{3} \alpha,
\end{aligned}
$$


and similarly $\operatorname{Pr}\left(c_{m} \mid s_{l}\right)=\frac{1}{3}, \operatorname{Pr}\left(c_{h} \mid s_{l}\right)=\frac{2}{3}(1-\alpha), \operatorname{Pr}\left(c_{l} \mid s_{h}\right)=\frac{2}{3}(1-\alpha), \operatorname{Pr}\left(c_{m} \mid s_{h}\right)=\frac{1}{3}$, and $\operatorname{Pr}\left(c_{h} \mid s_{h}\right)=\frac{2}{3} \alpha$. Furthermore, we have $\operatorname{Pr}\left(s_{l}\right)=\operatorname{Pr}\left(s_{h}\right)=1 / 2$.

U-supplier's expected cost conditional on each signal is given by

$$
C_{k}=c_{l} \operatorname{Pr}\left(c_{l} \mid s_{k}\right)+c_{m} \operatorname{Pr}\left(c_{m} \mid s_{k}\right)+c_{h} \operatorname{Pr}\left(c_{h} \mid s_{k}\right) \quad \text { for } k=l, h
$$

Substituting the posterior probabilities and cost levels into $C_{k}$, we have

$$
C_{l}=\mu-\frac{2}{3}(2 \alpha-1) \delta \text { and } C_{h}=\mu+\frac{2}{3}(2 \alpha-1) \delta .
$$

To model the correlation between U-suppliers' costs, we consider the following joint distributions of two U-suppliers' costs

$$
\begin{aligned}
& \operatorname{Pr}\left(\hat{c}_{i}=c_{k}, \hat{c}_{j}=c_{k}\right)=\tau, \quad \text { for } k=l, m, h, \\
& \operatorname{Pr}\left(\hat{c}_{i}=c_{k}, \hat{c}_{j}=c_{k^{\prime}}\right)=\frac{1}{6}-\frac{1}{2} \tau, \quad \text { for } k, k^{\prime}=l, m, h, k \neq k^{\prime},
\end{aligned}
$$

where $\tau \in(1 / 9,1 / 3]$. When $\tau=1 / 9$ the two U-suppliers' costs are independent, and when $\tau=1 / 3$ the costs are perfectly positively correlated. With the above joint distributions we can show that the marginal distribution for each U-supplier's cost follows the three-point distribution as specified at the beginning. Since $\operatorname{Cov}\left(\hat{c}_{i}, \hat{c}_{j}\right)=(3 \tau-1 / 3) \delta^{2}$ and $\operatorname{Corr}\left(\hat{c}_{i}, \hat{c}_{j}\right)=\frac{1}{2}(9 \tau-1)$, again we can use the parameter $\tau$ to measure the correlation between the U-suppliers' costs.

We assume U-suppliers' signals are conditionally independent given their costs. With the above specification for the joint cost distributions, we can calculate the joint distributions of the cost signals, as shown below:

$$
\begin{aligned}
& \operatorname{Pr}\left(s_{l}, s_{l}\right)=\operatorname{Pr}\left(s_{h}, s_{h}\right)=\frac{1}{6}(1+2 \alpha(1-\alpha))+3 \tau(\alpha-1 / 2)^{2}, \\
& \operatorname{Pr}\left(s_{l}, s_{h}\right)=\operatorname{Pr}\left(s_{h}, s_{l}\right)=\frac{1}{6}\left(1+\alpha^{2}+(1-\alpha)^{2}\right)-3 \tau(\alpha-1 / 2)^{2} .
\end{aligned}
$$

We can further define $\beta \equiv \frac{1}{6}(1+2 \alpha(1-\alpha))+3 \tau(\alpha-1 / 2)^{2}$. Then it can be shown that $1 / 4<\beta \leq 1 / 2$ for $\alpha \in(1 / 2,1]$ and $\tau \in(1 / 9,1 / 3]$.

Multi-point distributions: To illustrate how to incorporate multi-point distributions into our model, we consider a discrete uniform distribution with $n \geq 3$ cost levels denoted by $c_{1}, \cdots, c_{n}$ where $c_{1}<\cdots<c_{n}$. Let $\delta=\left(c_{n}-c_{1}\right) / 2$ and $\mu=\left(c_{n}+c_{1}\right) / 2$. Then the cost levels are

$$
c_{i}=\mu-\delta+\frac{2 \delta}{n-1}(i-1), \quad \text { for } i=1, \cdots, n .
$$

The probability of each cost level is $\operatorname{Pr}\left(\hat{c}=c_{i}\right)=1 / n$ for $i=1, \cdots, n$. Again we can use $\delta$ to indicate the extent of cost uncertainty.

Suppose the probabilities of signals conditional on costs are: for $i=1, \cdots, n$,

$$
\begin{aligned}
& \operatorname{Pr}\left(s_{l} \mid c_{i}\right)=\alpha-\frac{2 \alpha-1}{n-1}(i-1), \\
& \operatorname{Pr}\left(s_{h} \mid c_{i}\right)=1-\alpha+\frac{2 \alpha-1}{n-1}(i-1),
\end{aligned}
$$


where $\alpha \in(1 / 2,1]$ measures the signal quality or accuracy. Using Bayes' Theorem, we can calculate the posterior probabilities based on the above prior and conditional probabilities. Again we can show that $\operatorname{Pr}\left(s_{l}\right)=\operatorname{Pr}\left(s_{h}\right)=1 / 2$.

To model the correlation between U-suppliers' costs, we suppose the joint distributions of the two Usuppliers' costs are given by

$$
\begin{aligned}
\operatorname{Pr}\left(\hat{c}_{i}=c_{k}, \hat{c}_{j}=c_{k}\right) & =\tau, \quad \text { for } k=1, \cdots, n, \\
\operatorname{Pr}\left(\hat{c}_{i}=c_{k}, \hat{c}_{j}=c_{k^{\prime}}\right) & =\frac{1-n \tau}{n^{2}-n}, \quad \text { for } k, k^{\prime}=1, \cdots, n, k \neq k^{\prime},
\end{aligned}
$$

where $\tau \in\left(1 / n^{2}, 1 / n\right]$ measures the correlation between two U-suppliers' costs. When $\tau=1 / n^{2}$, the costs are independent, and when $\tau=1 / n$, the costs are perfectly correlated with each other. Similar to the two-point distribution, we can derive the joint distributions of the cost signals.

The above specifications imply that for multi-point distributions, we can use the same set of parameters (i.e., $\mu, \delta, \alpha, \tau)$ for the equilibrium analysis, and these parameters carry the same meaning as in the two-point distribution.

\section{Appendix D: Proofs of Main Results}

Proof of No Inventory Withholding Note that the contract terms selected in one supply chain are not observable to the firms in the other supply chain. First consider subgame uu. Suppose the manufacturers' optimal quantities out of the second-stage mechanism design are $q_{i l}, q_{j l}, q_{i h}$, and $q_{j h}$. Suppose the optimal selling quantity of manufacturer $i$, whose supplier has taken the contract with quantity $q_{i k}$, is $q_{i k}^{3}$ in the third stage, $k=l, h$ (note that $q_{i k}^{3}$ only depends on $k$ because the firms cannot observe the other chain's contracting terms).

Next, we argue that $q_{i k}^{3}<q_{i k}$ (withholding inventory) is impossible. When $q_{i k}^{3}<q_{i k}$, manufacturer $i$ can always improve her profit by decreasing $q_{i k}$ to $q_{i k}^{3}$. This is because manufacturer $i^{\prime} s$ second-stage mechanism design problem is formulated as follows:

$$
\begin{array}{ll}
\max _{q_{i l}, T_{i l}, q_{i h}, T_{i h}} \quad \beta\left[\left(A-q_{i l}^{3}-\gamma q_{j l}^{3}\right) q_{i l}^{3}-T_{i l}+\left(A-q_{i h}^{3}-\gamma q_{j h}^{3}\right) q_{i h}^{3}-T_{i h}\right] \\
+\left(\frac{1}{2}-\beta\right)\left[\left(A-q_{i l}^{3}-\gamma q_{j h}^{3}\right) q_{i l}^{3}-T_{i l}+\left(A-q_{i h}^{3}-\gamma q_{j l}^{3}\right) q_{i h}^{3}-T_{i h}\right] \\
=\frac{1}{2}\left(A-q_{i l}^{3}-\gamma\left(2 \beta q_{j l}^{3}+(1-2 \beta) q_{j h}^{3}\right)\right) q_{i l}^{3}-\frac{1}{2} T_{i l} \\
+\frac{1}{2}\left(A-q_{i h}^{3}-\gamma\left(2 \beta q_{j h}^{3}+(1-2 \beta) q_{j l}^{3}\right)\right) q_{i h}^{3}-\frac{1}{2} T_{i h}
\end{array}
$$

subject to the individual rationality and the incentive compatibility constraints:

$$
\begin{array}{ll}
\text { IRs: } & T_{i k}-C_{k} q_{i k} \geq 0, \forall k=l, h, \\
\text { ICs: } \quad T_{i k}-C_{k} q_{i k} \geq T_{i k^{\prime}}-C_{k} q_{i k^{\prime}}, \forall k, k^{\prime}=l, h \text { and } k \neq k^{\prime} .
\end{array}
$$

The revenue parts are determined by the third-stage selling quantities. But the transfer prices are determined by the contracting terms. By analyzing the IRs and ICs constraints, we derive $T_{i h}=C_{h} q_{i h}$ and $T_{i l}-C_{l} q_{i l}=T_{i h}-C_{l} q_{i h}$; that is, $T_{i h}=C_{h} q_{i h}$ and $T_{i l}=C_{l} q_{i l}+\left(C_{h}-C_{l}\right) q_{i h}$. This implies that $T_{i h}$ and $T_{i l}$ 
increase in $q_{i h}$ and $q_{i l}$. To reduce the transfer prices, manufacturer $i$ can decrease $q_{i h}$ and $q_{i l}$ to $q_{i h}^{3}$ and $q_{i l}^{3}$ respectively. That is, for any given $q_{j h}^{3}$ and $q_{j l}^{3}$, we must have $q_{i h}=q_{i h}^{3}$ and $q_{i l}=q_{i l}^{3}$, i.e., in equilibrium manufacturers will not withhold inventory.

Following similar arguments, we can show that under subgame $c u$, manufacturer $i$ and manufacturer $j$ will not order more than what they sell in the third stage either.

Proof of Proposition 1. First, for manufacturer $i$ 's problem in Equation (2), the objective function decreases in $T_{i}$. At optimality the constraint must be binding since otherwise manufacturer $i$ can always improve her profit by increasing the value of $T_{i}$. Thus, at optimality we have $T_{i}=c q_{i}$. Plugging this equation into $\Pi_{i}$ we rewrite manufacturer $i$ 's profit, $\Pi_{i}=\frac{1}{2}\left(A-q_{i}-\gamma q_{j l}\right) q_{i}+\frac{1}{2}\left(A-q_{i}-\gamma q_{j h}\right) q_{i}-c q_{i}$, which we can show is concave in $q_{i}$. Then from the first order condition we obtain manufacturer $i$ 's best response as follows,

$$
q_{i}\left(q_{j l}, q_{j h}\right)=\frac{A-c-\gamma\left(\frac{1}{2} q_{j l}+\frac{1}{2} q_{j h}\right)}{2} .
$$

Second, consider manufacturer $j$ 's problem in Equation (3). Note that the objective function decreases in $T_{j l}$ and $T_{j h}$. Combining IC(l) and IC(h) yields $q_{j l} \geq q_{j h}$. Since IR(h) and IC(l) together imply IR(l), IR(l) can be eliminated from the constraints. Next we show that if $\operatorname{IR}(\mathrm{h})$ and $\operatorname{IC}(\mathrm{l})$ are binding, then $\operatorname{IC}(\mathrm{h})$ will be automatically satisfied. To see this we obtain

$$
T_{j l}-C_{h} q_{j l}=T_{j h}-C_{l} q_{j h}+C_{l} q_{j l}-C_{h} q_{j l} \leq T_{j h}-C_{h} q_{j h},
$$

where the equality follows from the binding $\mathrm{IC}(\mathrm{l})$ and the inequality follows from $C_{h} q_{j h}-C_{l} q_{j h}+C_{l} q_{j l}-$ $C_{h} q_{j l}=\left(C_{h}-C_{l}\right)\left(q_{j h}-q_{j l}\right) \leq 0$. Therefore, for the optimal solution, $\operatorname{IR}(\mathrm{h})$ and $\operatorname{IC}(\mathrm{l})$ must be binding; thus we have $T_{j h}=C_{h} q_{j h}$ and $T_{j l}=C_{l} q_{j l}+\left(C_{h}-C_{l}\right) q_{j h}$. Then plugging these two equations into $\Pi_{j}$ we rewrite manufacturer $j$ 's objective function, $\Pi_{j}=\frac{1}{2}\left[\left(A-\gamma q_{i}-q_{j l}\right) q_{j l}-C_{l} q_{j l}\right]+\frac{1}{2}\left[\left(A-\gamma q_{i}-q_{j h}\right) q_{j h}-\left(2 C_{h}-C_{l}\right) q_{j h}\right]$. It is straightforward to show that $\Pi_{j}$ is jointly concave in $q_{j l}$ and $q_{j h}$. Thus, the first order conditions yield manufacturer $j$ 's best responses which are given by,

$$
q_{j l}\left(q_{i}\right)=\frac{A-C_{l}-\gamma q_{i}}{2}, \quad q_{j h}\left(q_{i}\right)=\frac{A-\gamma q_{i}-\left(2 C_{h}-C_{l}\right)}{2} .
$$

Solving the best responses of both manufacturers in Equations (D.1)-(D.2) yields the order quantities in equilibrium, $q_{i}^{c u}, q_{j l}^{c u}$, and $q_{j h}^{c u}$. The payments can be obtained by using the binding constraints, so we have $T_{i}^{c u}=c q_{i}^{c u}, T_{j l}^{c u}=C_{l} q_{j l}^{c u}+\left(C_{h}-C_{l}\right) q_{j h}^{c u}$, and $T_{j h}^{c u}=C_{h} q_{j h}^{c u}$. Finally, we calculate each player's profit by plugging the equilibrium quantities and payments into the objective functions.

Proof of Proposition 2. (i) Taking the first order derivative of $\Pi_{i}^{c u}$ with respect to $\delta$ we obtain $\frac{\partial \Pi_{i}^{c u}}{\partial \delta}=\frac{2 \gamma(2 \alpha-1)(2(A-c)-\gamma(A-\mu-(2 \alpha-1) \delta))}{\left(4-\gamma^{2}\right)^{2}}>0$ where the inequality follows from our assumption that $A>$ $\frac{2 c-\gamma(\mu+(2 \alpha-1) \delta)}{2-\gamma}$. Therefore, $\Pi_{i}^{c u}$ increases in $\delta$ for any $\delta$ satisfying the assumption. (ii) We take the first derivative of $\Pi_{j}^{c u}$ with respect to $\delta$, and obtain $\frac{\partial \Pi_{j}^{c u}}{\partial \delta}=-\frac{2(2 \alpha-1)\left(4(A-\mu)-2 \gamma(A-c)-\delta(2 \alpha-1)\left(\gamma^{4}-8 \gamma^{2}+20\right)\right)}{\left(4-\gamma^{2}\right)^{2}}$. We know from the assumption that $A>\frac{2 \mu+\left(6-\gamma^{2}\right)(2 \alpha-1) \delta-\gamma c}{2-\gamma}$ or $\delta<\frac{2(A-\mu)-\gamma(A-c)}{(2 \alpha-1)\left(6-\gamma^{2}\right)}$. Therefore, $\Pi_{j}^{c u}$ first decreases in $\delta$ when $\delta<\frac{2(2(A-\mu)-\gamma(A-c))}{(2 \alpha-1)\left(\gamma^{4}-8 \gamma^{2}+20\right)}$, and then increases in $\delta$ when $\delta>\frac{2(2(A-\mu)-\gamma(A-c))}{(2 \alpha-1)\left(\gamma^{4}-8 \gamma^{2}+20\right)}$. 
Proof of Proposition 3 (i) Since it is shown that $\frac{\partial q_{i}^{c u}}{\partial \alpha}=\frac{2 \gamma \delta}{4-\gamma^{2}}>0$ and $\Pi_{i}^{c u}=\left(q_{i}^{c u}\right)^{2}, \Pi_{i}^{c u}$ also increases in $\alpha$. (ii) Differentiating $\Pi_{j}^{c u}$ with respect to $\alpha$ we obtain

$$
\frac{\partial \Pi_{j}^{c u}}{\partial \alpha}=\frac{(2 \alpha-1)\left(\gamma^{4}-8 \gamma^{2}+20\right) \delta-4(A-\mu)+2 \gamma(A-c)}{\left(4-\gamma^{2}\right)^{2} /(4 \delta)} .
$$

The sign of $\frac{\partial \Pi_{j}^{c u}}{\partial \alpha}$ is the same with that of $\Omega \equiv(2 \alpha-1)\left(\gamma^{4}-8 \gamma^{2}+20\right) \delta-4(A-\mu)+2 \gamma(A-c)$. We can show that there exists a threshold of $\alpha, \hat{\alpha}_{m}=\frac{1}{2}+\frac{2(A-\mu)-\gamma(A-c)}{\delta\left(\gamma^{4}-8 \gamma^{2}+20\right)}$, such that $\Omega>0$ for $\alpha>\hat{\alpha}_{m}$ and $\Omega<0$ for $\alpha<\hat{\alpha}_{m}$. Thus, we conclude that $\frac{\partial \Pi_{j}^{c u}}{\partial \alpha}>0$ for $\alpha>\hat{\alpha}_{m}$ and $\frac{\partial \Pi_{j}^{c u}}{\partial \alpha}<0$ for $\alpha<\hat{\alpha}_{m}$.

Proof of Proposition 4. Similar to manufacturer $j$ 's problem in Equation (3) for subgame $c u$, we can show that $\operatorname{IR}(\mathrm{h})$ and $\mathrm{IC}(\mathrm{l})$ are binding, so we have $T_{i h}=C_{h} q_{i h}$ and $T_{i l}=C_{l} q_{i l}+\left(C_{h}-C_{l}\right) q_{i h}$. Plugging the two binding constraints into $\Pi_{i}$, we rewrite manufacturer $i$ 's objective function as follows:

$\Pi_{i}=\frac{1}{2}\left[\left(A-q_{i l}-\gamma\left(2 \beta q_{j l}+(1-2 \beta) q_{j h}\right)\right) q_{i l}-C_{l} q_{i l}-\left(C_{h}-C_{l}\right) q_{i h}\right]+\frac{1}{2}\left[\left(A-q_{i h}-\gamma\left((1-2 \beta) q_{j l}+2 \beta q_{j h}\right)\right) q_{i h}-C_{h} q_{i h}\right]$.

Solving the first order conditions yields manufacturer $i$ 's best responses:

$$
\begin{aligned}
q_{i l}\left(q_{j l}, q_{j h}\right) & =\frac{A-C_{l}-\gamma\left(2 \beta q_{j l}+(1-2 \beta) q_{j h}\right)}{2}, \\
q_{i h}\left(q_{j l}, q_{j h}\right) & =\frac{A-\left(2 C_{h}-C_{l}\right)-\gamma\left((1-2 \beta) q_{j l}+2 \beta q_{j h}\right)}{2} .
\end{aligned}
$$

Jointly solving the best responses for both manufacturers $i$ and $j$, we obtain the order quantities in equilibrium as shown in Equations (10)-(11). From the binding constraints we are able to get the equilibrium payments. Plugging the order quantities into $\Pi_{i}$ we obtain each manufacturer's equilibrium profit, $\Pi^{u u}=$ $\frac{1}{2}\left(q_{l}^{u u}\right)^{2}+\frac{1}{2}\left(q_{h}^{u u}\right)^{2}$. Each supplier's profit equals the expected information rent which is given by $\pi^{u u}=$ $\frac{1}{2}\left(C_{h}-C_{l}\right) q_{h}^{u u}$.

Proof of Corollary 1. The proof is straightforward, and thus is omitted.

Proof of Lemma 1. We have $\frac{\partial\left(\Delta_{2}-\Delta_{1}\right)}{\partial \tau}=\frac{32(2 \alpha-1)^{4} \gamma \delta^{2}}{\left(2+(4 \tau-1)(2 \alpha-1)^{2} \gamma\right)^{3}}>0$, so $\Delta_{2}-\Delta_{1}$ increases in $\tau$. In addition, we $\left.\left(\Delta_{2}-\Delta_{1}\right)\right|_{\tau=1 / 4}=\frac{4 \gamma(\mu+(2 \alpha-1) \delta-c)^{2}}{(2+\gamma)^{2}(2-\gamma)^{2}} \geq 0$. Then by the increase of $\Delta_{2}-\Delta_{1}$ in $\tau$, we have $\Delta_{2}>\Delta_{1}$ for $\tau \in(1 / 4,1 / 2]$.

Proof of Proposition 5. We have $\frac{\partial \Delta_{1}}{\partial c}=\frac{4(\gamma A-2 A-2 \gamma c+2 c+\gamma(\mu+(2 \alpha-1) \delta))}{\left(4-\gamma^{2}\right)^{2}}$. From the assumption $A>$ $\frac{2 c-\gamma(\mu+(2 \alpha-1) \delta)}{2-\gamma}$, we obtain $\frac{\partial \Delta_{1}}{\partial c}<\frac{8 \gamma(\mu+(2 \alpha-1) \delta-c)}{\left(\gamma^{2}-4\right)^{2}}$, which is no greater than 0 if $C_{h}=\mu+(2 \alpha-1) \delta \leq$ c. Moreover, $\left.\Delta_{1}\right|_{c=\mu+(2 \alpha-1) \delta}=-(2 \alpha-1)^{2} \delta^{2}<0$. Therefore, if $\mu+(2 \alpha-1) \delta \leq c, \Delta_{1}<0$ always holds as well. Now consider $\Delta_{2}$. We obtain $\frac{\partial \Delta_{2}}{\partial c}=\frac{4(-2 A+2 c+\gamma A-\gamma(\mu+(2 \alpha-1) \delta))}{\left(-4+\gamma^{2}\right)^{2}}$. Again from the assumption $A>$ $\frac{2 c-\gamma(\mu+(2 \alpha-1) \delta)}{2-\gamma}$ we show $\frac{\partial \Delta_{2}}{\partial c}<0$. Also we have $\left.\Delta_{2}\right|_{c=\mu+(2 \alpha-1) \delta}=-\frac{4(2 \alpha-1)^{2} \delta^{2}}{(2+(4 \beta-1) \gamma)^{2}}<0$. Therefore, if $\mu+(2 \alpha-$ 1) $\delta \leq c, \Delta_{2}<0$ always holds. In summary, when $c \geq \mu+(2 \alpha-1) \delta$, we have $\Delta_{1}<0$ and $\Delta_{2}<0$. Thus, the unique equilibrium is $\left(S_{u}, S_{u}\right)$. Next we prove the second part of the proposition for the case of $c \leq$ $\mu$. We have $\frac{\partial \Delta_{1}}{\partial A}=\frac{4(\mu+(2 \alpha-1) \delta-c)}{(2+\gamma)\left(4-\gamma^{2}\right)}>0$, for $c \leq \mu$. Also we obtain $\left.\Delta_{1}\right|_{A=\frac{2 \mu+(2 \alpha-1)\left(6-\gamma^{2}\right) \delta-\gamma c}{2-\gamma}}=\frac{\Gamma}{\left(4-\gamma^{2}\right)^{2}}$, where $\Gamma=-4(2 \alpha-1)\left(\gamma^{2}-6\right) \delta \mu-(1-2 \alpha)^{2}\left(\gamma^{4}-4 \gamma^{2}-4\right) \delta^{2}+4 c^{2}+4 c\left((2 \alpha-1)\left(\gamma^{2}-6\right) \delta-2 \mu\right)+4 \mu^{2}$. We have $\frac{\partial \Gamma}{\partial c}=4\left((2 \alpha-1)\left(\gamma^{2}-6\right) \delta+2 c-2 \mu\right)<0$. Also $\left.\Gamma\right|_{c=\mu}=\delta^{2}(2 \alpha-1)^{2}\left(4+4 \gamma^{2}-\gamma^{4}\right)>0$. Thus, $\Gamma>0$ for any

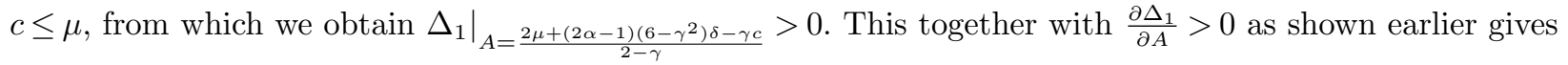
$\Delta_{1}>0$. Using the result $\Delta_{2}>\Delta_{1}$ in Lemma 1 , we obtain that, if $c \leq \mu$, then $\Delta_{1}>0$ and $\Delta_{2}>0$. Therefore, the unique equilibrium is $\left(S_{c}, S_{c}\right)$ in this case. 
Proof of Proposition 6. First we show $\frac{\partial \Delta_{1}}{\partial A}=\frac{\partial \Delta_{2}}{\partial A}=\frac{4(\mu+(2 \alpha-1) \delta-c)}{(2+\gamma)\left(4-\gamma^{2}\right)}>0$ for $c<C_{h}=\mu+(2 \alpha-1) \delta$. Moreover, we know from Lemma 1 that $\Delta_{2}>\Delta_{1}$ for $\mu<c<\mu+(2 \alpha-1) \delta$. Then it is straightforward to show that $A_{2}<A_{1}$. Finally, we can easily characterize the sourcing equilibria under different scenarios as shown in the proposition.

Proof of Proposition 7. We have $\frac{\partial A_{1}}{\partial \delta}=\frac{\Psi_{1}}{4(2-\gamma)(\mu+(2 \alpha-1) \delta-c)^{2}}$, where $\quad \Psi_{1}=(2 \alpha \quad-$ 1) $\left((2 \alpha-1) \delta\left(\gamma^{4}-8 \gamma^{2}+20\right)(2 \mu+(2 \alpha-1) \delta-2 c)+4(c-\mu)^{2}\right)$. Thus, the sign of $\frac{\partial A_{1}}{\partial \delta}$ is determined by $\Psi_{1}$. We have $\left.\Psi_{1}\right|_{\delta=(c-\mu) /(2 \alpha-1)}=-(2 \alpha-1)(c-\mu)^{2}\left(4-\gamma^{2}\right)^{2}<0$ for $\alpha \in(1 / 2,1],\left.\Psi_{1}\right|_{\delta=2(c-\mu) /(2 \alpha-1)}=4(c-\mu)^{2}>0$, and $\frac{\partial \Psi_{1}}{\partial \delta}=2(2 \alpha-1)^{2}(\mu+(2 \alpha-1) \delta-c)\left(20-8 \gamma^{2}+\gamma^{4}\right)>0$. Then, there must exist a threshold $\tilde{\delta} \in$ $((c-\mu) /(2 \alpha-1), 2(c-\mu) /(2 \alpha-1))$ such that $\Psi_{1}<0$ for $\delta<\tilde{\delta}$ and $\Psi_{1}>0$ for $\delta>\tilde{\delta}$. Since the sign of $\frac{\partial A_{1}}{\partial \delta}$ is the same as that of $\Psi_{1}$, we conclude that $\frac{\partial A_{1}}{\partial \delta} \leq 0$ for $\delta \leq \tilde{\delta}$ and $\frac{\partial A_{1}}{\partial \delta} \geq 0$ for $\delta \geq \tilde{\delta}$. That is, $A_{1}$ decreases in $\delta$ for $\delta \leq \tilde{\delta}$ and increases in $\delta$ for $\delta \geq \tilde{\delta}$. Similarly, $\frac{\partial A_{2}}{\partial \delta}=\frac{\Psi_{2}}{(2-\gamma)\left(2+(4 \tau-1)(2 \alpha-1)^{2} \gamma\right)^{2}(\mu+(2 \alpha-1) \delta-c)^{2}}$, where $\Psi_{2}$ is a long expression. First, evaluating $\Psi_{2}$ at $\delta=(c-\mu) /(2 \alpha-1)$, we have $\left.\Psi_{2}\right|_{\delta=(c-\mu) /(2 \alpha-1)}=-(2 \alpha-1)\left(4-\gamma^{2}\right)^{2}(c-$ $\mu)^{2}<0$ for $\alpha \in(1 / 2,1]$. Second, evaluating $\Psi_{2}$ at $\delta=2(c-\mu) /(2 \alpha-1)$, we have $\left.\Psi_{2}\right|_{\delta=2(c-\mu) /(2 \alpha-1)}=(2 \alpha-$ 1) $(1-\gamma)\left(2+(4 \tau-1)(2 \alpha-1)^{2} \gamma\right)^{2}(c-\mu)^{2} \geq 0$. Third, we obtain $\frac{\partial \Psi_{2}}{\partial \delta}=2(2 \alpha-1)^{2}(\mu+(2 \alpha-1) \delta-c)\left(M_{0}+\right.$ $\left.M_{1} \tau+M_{2} \tau^{2}\right)$, where $M_{0}=-8\left(2 \alpha^{2}-2 \alpha+1\right) \gamma+\left(16 \alpha^{4}-32 \alpha^{3}+40 \alpha^{2}-24 \alpha-3\right) \gamma^{2}-(2 \alpha-1)^{4} \gamma^{3}+\gamma^{4}+20$, $M_{1}=8(2 \alpha-1)^{2}(1-\gamma) \gamma\left(2-(2 \alpha-1)^{2} \gamma\right)$, and $M_{2}=16(2 \alpha-1)^{4}(1-\gamma) \gamma^{2} \geq 0$. Note that $M_{0}+M_{1} \tau+M_{2} \tau^{2}$ is a quadratic, convex function of $\tau$. The discriminant of $M_{0}+M_{1} \tau+M_{2} \tau^{2}$ is $-64(2 \alpha-1)^{4}(1-\gamma) \gamma^{2}\left(4-\gamma^{2}\right)^{2} \leq 0$. Therefore, $M_{0}+M_{1} \tau+M_{2} \tau^{2} \geq 0$, implying that $\frac{\partial \Psi_{2}}{\partial \delta} \geq 0$. Combining the above results, we conclude that there must exist a threshold $\hat{\delta} \in((c-\mu) /(2 \alpha-1), 2(c-\mu) /(2 \alpha-1)]$ such that $\Psi_{2}<0$ for $\delta<\hat{\delta}$ and $\Psi_{2}>0$ for $\delta>\hat{\delta}$. Since the sign of $\frac{\partial A_{2}}{\partial \delta}$ is the same as that of $\Psi_{2}$, we show $\frac{\partial A_{2}}{\partial \delta} \leq 0$ for $\delta \leq \hat{\delta}$ and $\frac{\partial A_{2}}{\partial \delta} \geq 0$ for $\delta \geq \hat{\delta}$. That is, $A_{2}$ decreases in $\delta$ for $\delta \leq \hat{\delta}$ and increases in $\delta$ for $\delta \geq \hat{\delta}$. Next, we compare the relative magnitude of $\tilde{\delta}$ and $\hat{\delta}$. We have $\Psi_{1}-\Psi_{2}=\gamma(2 \alpha-1)(\mu+(2 \alpha-1) \delta-c)^{2} \Psi_{3}$, where $\Psi_{3}$ is a long expression. Taking the derivative of $\Psi_{3}$ with respect to $\tau$ we obtain $\frac{\partial \Psi_{3}}{\partial \tau}=-8(2 \alpha-1)^{2}(1-\gamma)\left((2 \alpha-1)^{2} \gamma(4 \tau-1)+2\right) \leq 0$. Evaluating $\Psi_{3}$ at the upper limit of $\tau$ we obtain $\left.\Psi_{3}\right|_{\tau=1 / 2}=(2 \alpha-1)^{4} \gamma^{2}-\left(16 \alpha^{4}-32 \alpha^{3}+8 \alpha^{2}+8 \alpha+3\right) \gamma+16(1-\alpha) \alpha \geq 0$. Therefore, $\Psi_{3} \geq 0$ always holds, and hence $\Psi_{1}-\Psi_{2} \geq 0$. This, together with $\frac{\partial \Psi_{1}}{\partial \delta}>0, \frac{\partial \Psi_{2}}{\partial \delta}>0,\left.\Psi_{1}\right|_{\delta=\tilde{\delta}}=0$, and $\left.\Psi_{2}\right|_{\delta=\hat{\delta}}=0$, implies $\tilde{\delta} \leq \hat{\delta}$. Combining the results for $A_{1}$ and $A_{2}$ leads to Proposition 7 .

Proof of Proposition 8. We begin by examining the effect of $\alpha$ on $A_{1}$. Taking the derivative of $A_{1}$ with respect to $\alpha$ yields $\frac{\partial A_{1}}{\partial \alpha}=\frac{\delta \Omega_{1}}{2(2-\gamma)(\mu+(2 \alpha-1) \delta-c)^{2}}$, where $\Omega_{1}=(2 \alpha-1)\left(\gamma^{4}-8 \gamma^{2}+20\right) \delta(2 \mu+(2 \alpha-1) \delta-2 c)+$ $4(c-\mu)^{2}$. We observe the sign of $\frac{\partial A_{1}}{\partial \alpha}$ is the same as that of $\Omega_{1}$, so we will examine the sign of $\Omega_{1}$. First, taking derivative of $\Omega_{1}$ with respect to $\alpha$ yields $\frac{\partial \Omega_{1}}{\partial \alpha}=4\left(\gamma^{4}-8 \gamma^{2}+20\right)(\mu+(2 \alpha-1) \delta-c) \delta>0$ for $c<C_{h}=$ $\mu+(2 \alpha-1) \delta$, which indicates that $\Omega_{1}$ is increasing in $\alpha$. Second, evaluating $\Omega_{1}$ at the lower bound of $\alpha$ yields $\left.\Omega_{1}\right|_{\alpha=1 / 2+(c-\mu) /(2 \delta)}=-\left(4-\gamma^{2}\right)^{2}(c-\mu)^{2}<0$. Third, evaluating $\Omega_{1}$ at the upper bound of $\alpha$ yields $\left.\Omega_{1}\right|_{\alpha=1}=4(c-\mu)^{2}-2 M \delta(c-\mu)+M \delta^{2}$, where we define $M=20-8 \gamma^{2}+\gamma^{4}$ for $\gamma \in[0,1]$. Note that $\left.\Omega_{1}\right|_{\alpha=1}$ is a quadratic function of $c-\mu$, and $c-\mu<(2 \alpha-1) \delta \leq \delta$. We can show that $\left.\Omega_{1}\right|_{\alpha=1}>0$ if $(c-\mu) / \delta<$ $\frac{M-\sqrt{M^{2}-4 M}}{4}$ and $\left.\Omega_{1}\right|_{\alpha=1}<0$ if $(c-\mu) / \delta>\frac{M-\sqrt{M^{2}-4 M}}{4}$. Combining the above results together, we obtain: (1) if $(c-\mu) / \delta<\frac{M-\sqrt{M^{2}-4 M}}{4}$, then there exists a threshold of $\alpha, \tilde{\alpha} \in(1 / 2+(c-\mu) /(2 \delta), 1)$, such that $\Omega_{1}<0$ for $\alpha<\tilde{\alpha}$ and $\Omega_{1}>0$ for $\alpha>\tilde{\alpha} ;(2)$ if $(c-\mu) / \delta>\frac{M-\sqrt{M^{2}-4 M}}{4}$, then $\Omega_{1}<0$ holds for any $\alpha$. 
Now consider the effect of $\alpha$ on $A_{2}$. To move the analysis forward, we focus on the case where $\tau=1 / 4$. Taking the derivative of $A_{2}$ with respect to $\alpha$ yields $\frac{\partial A_{2}}{\partial \alpha}=\frac{\delta \Omega_{2}}{2(2-\gamma)(\mu+(2 \alpha-1) \delta-c)^{2}}$, where $\Omega_{2}$ is a long expression. The sign of $\frac{\partial A_{2}}{\partial \alpha}$ is the same as that of $\Omega_{2}$. First, taking derivative of $\Omega_{2}$ with respect to $\alpha$ yields $\frac{\partial \Omega_{2}}{\partial \alpha}=$ $4\left(\gamma^{4}-8 \gamma^{2}-4 \gamma+20\right)(\mu+(2 \alpha-1) \delta-c) \delta>0$ for $c<\mu+(2 \alpha-1) \delta$, which indicates that $\Omega_{2}$ is increasing in $\alpha$. Second, evaluating $\Omega_{2}$ at the lower bound of $\alpha$ yields $\left.\Omega_{2}\right|_{\alpha=1 / 2+(c-\mu) /(2 \delta)}=-\left(4-\gamma^{2}\right)^{2}(c-\mu)^{2}<0$. Third, evaluating $\Omega_{2}$ at the upper bound of $\alpha$ yields $\left.\Omega_{2}\right|_{\alpha=1}=4(1-\gamma)(c-\mu)^{2}-2 N \delta(c-\mu)+N \delta^{2}$, where we define $N=20-4 \gamma-8 \gamma^{2}+\gamma^{4}$ for $\gamma \in[0,1]$. Again $\left.\Omega_{2}\right|_{\alpha=1}$ is a quadratic function of $c-\mu$, and $c-\mu<(2 \alpha-1) \delta \leq \delta$. We can show that $\left.\Omega_{2}\right|_{\alpha=1}>0$ if $(c-\mu) / \delta<\frac{N-\sqrt{N^{2}-4(1-\gamma) N}}{4(1-\gamma)}$ and $\left.\Omega_{2}\right|_{\alpha=1}<0$ if $(c-\mu) / \delta>\frac{N-\sqrt{N^{2}-4(1-\gamma) N}}{4(1-\gamma)}$. Combining the above results together, we obtain: (1) if $(c-\mu) / \delta<\frac{N-\sqrt{N^{2}-4(1-\gamma) N}}{4(1-\gamma)}$, then there exists a threshold of $\alpha, \hat{\alpha} \in(1 / 2+(c-\mu) /(2 \delta), 1)$, such that $\Omega_{2}<0$ for $\alpha<\hat{\alpha}$ and $\Omega_{2}>0$ for $\alpha>\hat{\alpha} ;(2)$ if $(c-\mu) / \delta>$ $\frac{N-\sqrt{N^{2}-4(1-\gamma) N}}{4(1-\gamma)}$, then $\Omega_{2}<0$ holds for any $\alpha$.

Next we compare $\tilde{\alpha}$ and $\hat{\alpha}$. First we observe $\left.\Omega_{1}\right|_{\alpha=1 / 2+(c-\mu) /(2 \delta)}=\left.\Omega_{2}\right|_{\alpha=1 / 2+(c-\mu) /(2 \delta)}$. Furthermore, $\Omega_{1}-$ $\Omega_{2}=4 \gamma(-2 \alpha \delta+c+\delta-\mu)^{2}>0$. This, together with $\frac{\partial \Omega_{1}}{\partial \alpha}>0$ and $\frac{\partial \Omega_{2}}{\partial \alpha}>0$, implies that $\tilde{\alpha}<\hat{\alpha}$. We also obtain $\frac{M-\sqrt{M^{2}-4 M}}{4}>\frac{N-\sqrt{N^{2}-4(1-\gamma) N}}{4(1-\gamma)}$ for $\gamma \in[0,1]$.

Finally, since the sign of $\frac{\partial A_{1}}{\partial \alpha}\left(\frac{\partial A_{2}}{\partial \alpha}\right)$ is the same as that of $\Omega_{1}\left(\Omega_{2}\right)$, we can derive the results in the proposition.

Proof of Proposition 9. (i) Regarding $A_{1}$ we obtain $\frac{\partial A_{1}}{\partial \gamma}=\frac{\Gamma_{1}}{4(2-\gamma)^{2}(\mu+(2 \alpha-1) \delta-c)}$, where $\Gamma_{1}=4(\mu+(2 \alpha-$ 1) $\delta-c)^{2}-(2 \alpha-1)^{2}\left(3 \gamma^{4}-8 \gamma^{3}-8 \gamma^{2}+32 \gamma-16\right) \delta^{2}$. Thus, the sign of $\frac{\partial A_{1}}{\partial \gamma}$ is the same as that of $\Gamma_{1}$. We have $\frac{\partial \Gamma_{1}}{\partial \gamma}=-4(2 \alpha-1)^{2} \delta^{2}(2-\gamma)\left(4-3 \gamma^{2}\right)<0$ for $\gamma \in[0,1]$. Evaluating $\Gamma_{1}$ at $\gamma=0$, we obtain $\left.\Gamma_{1}\right|_{\gamma=0}=$ $4(c-\mu)^{2}+8(2 \alpha-1) \delta\left(\mu+\frac{5}{2}(2 \alpha-1) \delta-c\right)>0$ for $c \in\left(\mu, C_{h}=\mu+(2 \alpha-1) \delta\right)$. Evaluating $\Gamma_{1}$ at $\gamma=1$, we obtain $\left.\Gamma_{1}\right|_{\gamma=1}=4(\mu+(2 \alpha-1) \delta-c)^{2}-3(2 \alpha-1)^{2} \delta^{2}$, whose sign depends on $c$. We consider two cases: (1) If $c \leq \mu+(1-\sqrt{3} / 2)(2 \alpha-1) \delta$, then $\left.\Gamma_{1}\right|_{\gamma=1} \geq 0$; (2) if $c>\mu+(1-\sqrt{3} / 2)(2 \alpha-1) \delta$, then $\left.\Gamma_{1}\right|_{\gamma=1}<0$. Combining the above results, we can conclude that: (1) if $c \leq \mu+(1-\sqrt{3} / 2)(2 \alpha-1) \delta$, then $\Gamma_{1} \geq 0$ for $\gamma \in[0,1]$, and thus $\frac{\partial A_{1}}{\partial \gamma} \geq 0$ always holds; (2) if $c>\mu+(1-\sqrt{3} / 2)(2 \alpha-1) \delta$, then there exists a threshold of $\gamma, \tilde{\gamma} \in(0,1)$, such that $\frac{\partial A_{1}}{\partial \gamma}>0$ if $\gamma<\tilde{\gamma}$, and $\frac{\partial A_{1}}{\partial \gamma}<0$ if $\gamma>\tilde{\gamma}$. That is, in this case, $A_{1}$ first increases and then decreases in $\gamma$. (ii) Now consider the effect of $\gamma$ on $A_{2}$. We obtain $\frac{\partial A_{2}}{\partial \gamma}=\frac{\Gamma_{2}}{(2-\gamma)^{2}\left(2+(4 \tau-1)(2 \alpha-1)^{2} \gamma\right)^{3}(c-\mu-(2 \alpha-1) \delta)}$, where $\Gamma_{2}$ is a long expression, and the sign of $\frac{\partial A_{2}}{\partial \gamma}$ is opposite to that of $\Gamma_{2}$. It has been shown that $\Gamma_{2}$ is concave in $\gamma$. Evaluating $\Gamma_{2}$ at $\gamma=1$, we find that $\left.\Gamma_{2}\right|_{\gamma=1}$ is a convex, quadratic function of $c$. Also the discriminant of $\left.\Gamma_{2}\right|_{\gamma=1}$ is $-12(2 \alpha-1)^{2} \delta^{2}\left((2 \alpha-1)^{2}(4 \tau-1)+2\right)^{3}\left(7(2 \alpha-1)^{2}(4 \tau-1)+2\right)<$ 0. Therefore, $\left.\Gamma_{2}\right|_{\gamma=1}>0$ always holds. Now evaluating $\Gamma_{2}$ at $\gamma=0$, we obtain $\left.\Gamma_{2}\right|_{\gamma=0}=$ $8\left((\delta-2 \alpha \delta)^{2}\left(32 \alpha^{2}(4 \tau-1)-32 \alpha(4 \tau-1)+32 \tau-11\right)+2(2 \alpha-1) \delta \mu+\mu^{2}\right)+8 c^{2}-16 c((2 \alpha-1) \delta+\mu)$, which is a convex, quadratic function of $c$, and its discriminant equals $1024(2 \alpha-1)^{2} \delta^{2}\left(1-2(4 \tau-1)(2 \alpha-1)^{2}\right)$. Therefore, if $\tau \geq \frac{1}{4}+\frac{1}{8(2 \alpha-1)^{2}}$, the discriminant is no greater than zero and hence $\left.\Gamma_{2}\right|_{\gamma=0} \geq 0$. If $\tau<\frac{1}{4}+\frac{1}{8(2 \alpha-1)^{2}}$, setting $\left.\Gamma_{2}\right|_{\gamma=0}=0$ leads to $c=\mu+(2 \alpha-1) \delta \pm 2(2 \alpha-1) \delta \sqrt{1-2(4 \tau-1)(2 \alpha-1)^{2}}$. By $c<C_{h}=\mu+(2 \alpha-1) \delta$ and the convexity of $\left.\Gamma_{2}\right|_{\gamma=0}$ in $c$, we have $\left.\Gamma_{2}\right|_{\gamma=0} \geq 0$ for $c \leq \mu+(2 \alpha-1) \delta-2(2 \alpha-1) \delta \sqrt{1-2(4 \tau-1)(2 \alpha-1)^{2}}$ and $\left.\Gamma_{2}\right|_{\gamma=0}<0$ for $c>\mu+(2 \alpha-1) \delta-2(2 \alpha-1) \delta \sqrt{1-2(4 \tau-1)(2 \alpha-1)^{2}}$. To summarize, If $\tau \geq \frac{1}{4}+\frac{1}{8(2 \alpha-1)^{2}}$, then $\left.\Gamma_{2}\right|_{\gamma=0} \geq 0$ and $\left.\Gamma_{2}\right|_{\gamma=1}>0$, and thus $\Gamma_{2} \geq 0$ always holds by the concavity of $\Gamma_{2}$ in $\gamma$; in this case, $A_{2}$ decreases in $\gamma$. If $\tau<\frac{1}{4}+\frac{1}{8(2 \alpha-1)^{2}}$ and $c \leq \mu+(2 \alpha-1) \delta-2(2 \alpha-1) \delta \sqrt{1-2(4 \tau-1)(2 \alpha-1)^{2}}$, again $\Gamma_{2} \geq 0$ always holds, 
and hence $A_{2}$ decreases in $\gamma$. If $\tau<\frac{1}{4}+\frac{1}{8(2 \alpha-1)^{2}}$ and $c>\mu+(2 \alpha-1) \delta-2(2 \alpha-1) \delta \sqrt{1-2(4 \tau-1)(2 \alpha-1)^{2}}$, then $\left.\Gamma_{2}\right|_{\gamma=0}<0$ and $\left.\Gamma_{2}\right|_{\gamma=1}>0$; by the concavity of $\Gamma_{2}$ in $\gamma$, there exists a threshold $\hat{\gamma} \in(0,1)$ such that $\Gamma_{2}<0$ for $\gamma<\hat{\gamma}$ and $\Gamma_{2} \geq 0$ for $\gamma \geq \hat{\gamma}$; in this case, $A_{2}$ first increases and then decreases in $\gamma$.

Proof of Proposition 10. Since $A>A_{1}$, the current equilibrium is $\left(S_{c}, S_{c}\right)$. Suppose the value of $c$ increases by $\kappa$. Then, we use the superscript " $\kappa$ " to denote the thresholds and expected profits after the increase of $c$ by $\kappa \cdot A_{1}^{\kappa}=\mu+(2 \alpha-1) \delta+\frac{9 \delta^{2}}{4(\mu+(2 \alpha-1) \delta-c-\kappa)}$. In the following, we first show that $\kappa>\underline{\kappa}$ implies $A<A_{1}^{\kappa} . A<A_{1}^{\kappa}$ is equivalent to $\kappa>\mu+(2 \alpha-1) \delta-c-\frac{9(2 \alpha-1)^{2} \delta^{2}}{4(A-\mu-(2 \alpha-1) \delta)} \equiv \kappa_{1}$. If $\underline{\kappa}>\kappa_{1}$, then $\kappa>\underline{\kappa}$ implies $\kappa>$ $\kappa_{1}$ (i.e., $\left.A<A_{1}^{\kappa}\right)$. Now we show that $\underline{\kappa}>\kappa_{1} \cdot \underline{\kappa}-\kappa_{1}=\sqrt{(A-c)^{2}-9(2 \alpha-1)^{2} \delta^{2}}-(A+c-2 \mu-2(2 \alpha-1) \delta)-$ $\left(\mu+(2 \alpha-1) \delta-c-\frac{9(2 \alpha-1)^{2} \delta^{2}}{4(A-\mu-(2 \alpha-1) \delta)}\right)=\sqrt{(A-c)^{2}-9 \delta^{2}}+\frac{9(2 \alpha-1)^{2} \delta^{2}}{4(A-\mu-(2 \alpha-1) \delta)}-(A-\mu-(2 \alpha-1) \delta)$. By $A>$ $A_{1}=\mu+(2 \alpha-1) \delta+\frac{9(2 \alpha-1)^{2} \delta^{2}}{4(\mu+(2 \alpha-1) \delta-c)}$, we have $c<\mu+(2 \alpha-1) \delta-\frac{9(2 \alpha-1)^{2} \delta^{2}}{4(A-\mu-(2 \alpha-1) \delta)}$. Thus $\underline{\kappa}-\kappa_{1}>$ $\sqrt{\left(A-\left(\mu+(2 \alpha-1) \delta-\frac{9(2 \alpha-1)^{2} \delta^{2}}{4(A-\mu-(2 \alpha-1) \delta)}\right)\right)^{2}-9(2 \alpha-1)^{2} \delta^{2}}+\frac{9(2 \alpha-1)^{2} \delta^{2}}{4(A-\mu-(2 \alpha-1) \delta)}-(A-\mu-(2 \alpha-1) \delta)=A-\mu-$ $(2 \alpha-1) \delta-\frac{9(2 \alpha-1)^{2} \delta^{2}}{4(A-\mu-(2 \alpha-1) \delta)}+\frac{9(2 \alpha-1)^{2} \delta^{2}}{4(A-\mu-(2 \alpha-1) \delta)}-(A-\mu-(2 \alpha-1) \delta)=0$. That is, $\underline{\kappa}>\kappa_{1}$ indeed holds, and $\kappa>\underline{\kappa}$ implies $A<A_{1}^{\kappa}$. Next, we show that $A>A_{2}^{\kappa}$ is implied by $\kappa<\bar{\kappa} . A_{2}^{\kappa}=c+\kappa+\frac{9(2 \alpha-1)^{2} \delta^{2}}{(1+4 \beta)^{2}(\mu+(2 \alpha-1) \delta-c-\kappa)}$. $A>A_{2}^{\kappa}$ is equivalent to $\kappa<(A+\mu+(2 \alpha-1) \delta-2 c) / 2-\sqrt{(A-\mu-(2 \alpha-1) \delta)^{2} / 4+9(2 \alpha-1)^{2} \delta^{2} /(1+4 \beta)^{2}}$, which is guaranteed by $\kappa<\bar{\kappa}$. Since $A_{1}<A$ and $A_{2}^{\kappa}<A<A_{1}^{\kappa}$, the sourcing equilibrium will shift from $\left(S_{c}, S_{c}\right)$ to $\left(S_{c}, S_{u}\right)$. Without losing the generality, suppose manufacturer 2 switches from the Csupplier to U-supplier. Then manufacturer 2's profit changes by $\Pi_{2}^{c u \kappa}-\Pi_{2}^{c c}=\frac{1}{2}\left(\frac{A-\mu+(2 \alpha-1) \delta}{3}+\frac{c+\kappa-\mu}{3}\right)^{2}+$ $\frac{1}{2}\left(\frac{A-\mu-(2 \alpha-1) \delta}{3}+\frac{c+\kappa-\mu-4(2 \alpha-1) \delta}{3}\right)^{2}-\left(\frac{A-c}{3}\right)^{2} \cdot \Pi_{2}^{c u \kappa}-\Pi_{2}^{c c}>0$ leads to $\kappa>\underline{\kappa}$. Manufacturer 1 still sources from $S_{c}$, and manufacturer 1's profit changes by $\Pi_{1}^{c u \kappa}-\Pi_{1}^{c c}=\left(\frac{A-c-\kappa}{3}+\frac{\mu+(2 \alpha-1) \delta-c-\kappa}{3}\right)^{2}-\left(\frac{A-c}{3}\right)^{2} . \Pi_{1}^{c u \kappa}-\Pi_{1}^{c c}>0$ leads to $\kappa<\frac{\mu+(2 \alpha-1) \delta-c}{2}$, which is guaranteed by $\kappa<\bar{\kappa}$. Therefore, if $\underline{\kappa}<\kappa<\bar{\kappa}$, the sourcing equilibrium will shift from $\left(S_{c}, S_{c}\right)$ to $\left(S_{c}, S_{u}\right)$ and both manufacturers are better off.

\section{Appendix E: Proofs of Results in Appendices A and B}

Proof of Lemma A.1. Since manufacturer $i$ 's problem is the same as Equation (2), manufacturer $i$ 's best response is the same as Equation (D.1). Now consider manufacturer $j$ 's problem:

$$
\max _{q_{j l}, T_{j l}, q_{j h}, T_{j h} \geq 0} \Pi_{j}=\frac{1}{2}\left[\left(A-\gamma q_{i}-q_{j l}\right) q_{j l}-T_{j l}\right]+\frac{1}{2}\left[\left(A-\gamma q_{i}-q_{j h}\right) q_{j h}-T_{j h}\right],
$$

subject to $T_{j l}-C_{l} q_{j l} \geq 0$ and $T_{j h}-C_{h} q_{j h} \geq 0$. Since $\Pi_{j}$ decreases in $T_{j l}$ and $T_{j h}$, the constraints must be binding. Then plugging the binding constraints into $\Pi_{j}$, we rewrite the objective function as $\Pi_{j}=$ $\frac{1}{2}\left[\left(A-\gamma q_{i}-q_{j l}\right) q_{j l}-C_{l} q_{j l}\right]+\frac{1}{2}\left[\left(A-\gamma q_{i}-q_{j h}\right) q_{j h}-C_{h} q_{j h}\right]$. Solving the first order conditions we obtain manufacturer $j$ ' best responses as follows:

$$
q_{j l}\left(q_{i}\right)=\frac{A-C_{l}-\gamma q_{i}}{2}, \quad q_{j h}\left(q_{i}\right)=\frac{A-C_{h}-\gamma q_{i}}{2} .
$$

Jointly solving the three best responses in Equations (D.1) and (E.1) we obtain the equilibrium quantities, $\bar{q}_{i}^{c u}, \bar{q}_{j l}^{c u}, \bar{q}_{j h}^{c u}$, as shown in the lemma. Using the binding constraints, we can calculate the equilibrium payments. Then the equilibrium profits for both manufacturers follow, and each supplier makes zero profit. 
Proof of Lemma A.2. First, since $\Pi_{i}$ increases in $T_{i l}$ and $T_{i h}$, the constraints must be binding for the optimal solution. So we have $T_{i l}=C_{l} q_{i l}$ and $T_{i h}=C_{h} q_{i h}$. Plugging these two equations into manufacturer $i$ 's objective function, we obtain $\Pi_{i}=\frac{1}{2}\left[\left(A-q_{i l}-\gamma\left(2 \beta q_{j l}+(1-2 \beta) q_{j h}\right)\right) q_{i l}-C_{l} q_{i l}\right]+\frac{1}{2}\left[\left(A-q_{i h}-\gamma\left((1-2 \beta) q_{j l}+\right.\right.\right.$ $\left.\left.\left.2 \beta q_{j h}\right)\right) q_{i h}-C_{h} q_{i h}\right]$. Then it is easy to show that $\Pi_{i}$ is jointly concave in $q_{i l}$ and $q_{i h}$. Thus, from the first order conditions we obtain manufacturer $i$ 's best responses as follows:

$$
q_{i l}\left(q_{j l}, q_{j h}\right)=\frac{A-C_{l}-\gamma\left[2 \beta q_{j l}+(1-2 \beta) q_{j h}\right]}{2}, \quad q_{i h}\left(q_{j l}, q_{j h}\right)=\frac{A-C_{h}-\gamma\left[(1-2 \beta) q_{j l}+2 \beta q_{j h}\right]}{2} .
$$

By jointly solving the above best responses for both manufacturers, we obtain the symmetric BNE $\left(\bar{q}_{l}^{u u}, \bar{T}_{l}^{u u}, \bar{q}_{h}^{u u}, \bar{T}_{h}^{u u}\right)$ as shown in the lemma. The payments $\bar{T}_{l}^{u u}$ and $\bar{T}_{h}^{u u}$ are obtained from the binding constraints. Then we show that the equilibrium profit of each manufacturer is $\bar{\Pi}^{u u}=\frac{1}{2}\left(\bar{q}_{l}^{u u}\right)^{2}+\frac{1}{2}\left(\bar{q}_{h}^{u u}\right)^{2}$. Each supplier makes zero profit.

Proof of Proposition A.1. To derive the equilibrium for the case with symmetric information we need to compare $\bar{\Pi}^{c c}$ with $\bar{\Pi}_{1}^{u c}$, and $\bar{\Pi}_{1}^{c u}$ with $\bar{\Pi}^{u u}$. Define $\bar{\Delta}_{1}=\Pi^{c c}-\bar{\Pi}_{2}^{c u}$ and $\bar{\Delta}_{2}=\bar{\Pi}_{1}^{c u}-\bar{\Pi}^{u u}$. We have $\frac{\partial\left(\bar{\Delta}_{1}-\bar{\Delta}_{2}\right)}{\partial \tau}=$ $-\frac{8(2 \alpha-1)^{4} \delta^{2} \gamma}{\left(2+(4 \tau-1)(2 \alpha-1)^{2} \gamma\right)^{3}}<0$. Also $\left.\left(\bar{\Delta}_{1}-\bar{\Delta}_{2}\right)\right|_{\tau=1 / 4}=\frac{-4(c-\mu)^{2} \gamma}{(\gamma-2)^{2}(\gamma+2)^{2}}$. Therefore, when $\tau \in(1 / 4,1 / 2]$, we have $\bar{\Delta}_{2}>$ $\bar{\Delta}_{1}$. Now consider the case where $\mu \leq c$. Taking the first order derivative of $\bar{\Delta}_{1}$ with respect to $\mu$, we obtain $\frac{\partial \bar{\Delta}_{1}}{\partial \mu}=\frac{4(2 A-2 \mu+\gamma c-\gamma A)}{\left(4-\gamma^{2}\right)^{2}}>0$. Moreover, $\left.\bar{\Delta}_{1}\right|_{\mu=c}=-(2 \alpha-1)^{2} \delta^{2} / 4<0$. Therefore, $\bar{\Delta}_{1}<0$ for $\mu \leq c$. Similarly, we have $\frac{\partial \bar{\Delta}_{2}}{\partial \mu}=-\frac{4(A(\gamma-2)+c \gamma-2(\gamma-1) \mu)}{\left(\gamma^{2}-4\right)^{2}} \geq-\frac{4(A(\gamma-2)+A \gamma-2(\gamma-1) \mu)}{\left(\gamma^{2}-4\right)^{2}}=-\frac{4(A-\mu)}{(\gamma-2)(\gamma+2)^{2}}>0$, where the inequality follows from the assumption that $A>c$. Furthermore, $\left.\bar{\Delta}_{2}\right|_{\mu=c}=-\frac{(2 \alpha-1)^{2} \delta^{2}}{\left(\left(2+(2 \alpha-1)^{2}(4 \beta-1) \gamma\right)^{2}\right.}<0$. Thus, $\bar{\Delta}_{2}<0$ for $\mu \leq c$. Combining the above results, we can show that the unique equilibrium for the sourcing game is $\left(S_{u}, S_{u}\right)$.

Proof of Proposition B.1. Note the assumption $\delta<(2-\gamma+4 \gamma \beta)(A-\mu) /((2 \alpha-1)(4 \gamma \beta+\gamma+6))$. Evaluating $\frac{\partial \Pi^{u u}}{\partial \delta}$ at the upper bound of $\delta$, we obtain

$$
\left.\frac{\partial \Pi^{u u}}{\partial \delta}\right|_{\delta=\frac{(2-\gamma+4 \gamma \beta)(A-\mu)}{(2 \alpha-1)(4 \gamma \beta+\gamma+6)}}=\frac{4(2 \alpha-1)(A-\mu)(2+3 \gamma-4 \beta \gamma)}{(\gamma+2)(4 \beta \gamma+\gamma+6)((4 \beta-1) \gamma+2)}>0,
$$

for $\beta \in\left(\frac{1}{4}, \frac{1}{2}\right], \alpha \in(1 / 2,1]$ and $\gamma \in[0,1]$. We also know that $\frac{\partial^{2} \Pi^{u u}}{\partial \delta^{2}}=\frac{(2 \alpha-1)^{2}\left((2+3 \gamma-4 \gamma \beta)^{2}+(6+\gamma+4 \gamma \beta)^{2}\right)}{\left(4-\gamma^{2}+4 \gamma^{2} \beta+8 \gamma \beta\right)^{2}}>0$. Thus, $\Pi^{u u}$ is convex in $\delta$. Setting $\frac{\partial \Pi^{u u}}{\partial \delta}=0$, we obtain $\delta=\frac{(A-\mu)((4 \beta-1) \gamma+2)^{2}}{(2 \alpha-1)\left(\left(16 \beta^{2}-8 \beta+5\right) \gamma^{2}+4(4 \beta+3) \gamma+20\right)} \equiv \delta_{m}^{u u}$. Therefore, $\Pi^{u u}$ first decreases in $\delta$ when $\delta<\delta_{m}^{u u}$, and then increases in $\delta$ when $\delta>\delta_{m}^{u u}$.

Proof of Proposition B.2. Taking the derivative of $\Pi^{u u}$ with respect to $\alpha$, we have $\frac{\partial \Pi^{u u}}{\partial \alpha}=$ $-\frac{A-\mu-4 \delta\left((2 \alpha-1)\left(\gamma^{2}+4 \gamma+5\right) \delta\right)}{(\gamma+2)^{2}}$. It can be readily shown that $\frac{\partial \Pi^{u u}}{\partial \alpha}$ increases in $\alpha$. Evaluating $\frac{\partial \Pi^{u u}}{\partial \alpha}$ at $\alpha=1 / 2$, we obtain $\left.\frac{\partial \Pi^{u u}}{\partial \alpha}\right|_{\alpha=1 / 2}=-\frac{4 \delta(A-\mu)}{(\gamma+2)^{2}}<0$. Evaluating $\frac{\partial \Pi^{u u}}{\partial \alpha}$ at $\alpha=\frac{A+\gamma \delta+3 \delta-\mu}{2(\gamma+3) \delta}$ we obtain $\left.\frac{\partial \Pi^{u u}}{\partial \alpha}\right|_{\alpha=\frac{A+\gamma \delta+3 \delta-\mu}{2(\gamma+3) \delta}}=$ $\frac{4(\gamma+1) \delta(A-\mu)}{(\gamma+2)(\gamma+3)}>0$. Therefore, there must exist a threshold of $\alpha, \alpha_{m}^{u u}=\frac{A+\left(\gamma^{2}+4 \gamma+5\right) \delta-\mu}{2\left(\gamma^{2}+4 \gamma+5\right) \delta}$, such that $\frac{\partial \Pi^{u u}}{\partial \alpha}<0$ for $\alpha<\alpha_{m}^{u u}$ and $\frac{\partial \Pi^{u u}}{\partial \alpha}>0$ for $\alpha>\alpha_{m}^{u u}$. Therefore, the manufacturer's profit first decreases and then increases in $\alpha$. 